\title{
El análisis de la calidad del empleo a partir de un índice multidimensional: una mirada al mercado de trabajo urbano en Argentina (2003 y 2015)*
}

\section{The analysis of the quality of employment based on a multidimensional index: a look at the urban labor market in Argentina (2003-2015)}

\author{
Diego Born ${ }^{* *}$ y Nicolás Sacco ${ }^{* * *}$
}

\section{Resumen}

El artículo presenta la metodología de construcción de un índice multidimensional que resume las diferentes situaciones laborales en las que se encuentran aquellos que conforman la población económicamente activa. Para ejemplificar la aplicación y potencia de esta herramienta, se analizó la dinámica del mercado de trabajo urbano argentino durante el período 2003-2015.

El Índice de Situación del Mercado Laboral Urbano (IMSLU)fue construido a partir de la información disponible en las bases de la Encuesta Permanente de Hogares del Instituto Nacional de Estadística y Censos. Condensa variables que dan cuenta de diversos aspectos del mercado de trabajo y se compone de dos grandes dimensiones: precariedad e ingresos. Se trata de una

\footnotetext{
* Artículo recibido 4 de abril de 2016. Aceptado 4 de octubre de 2016.

Este trabajo constituye una versión corregida y extendida en el tiempo de dos ponencias de 2012 presentadas en las V Jornadas de Economía Crítica, Facultad de Ciencias Económicas de la Universidad de Buenos Aires y en las VII Jornadas de Sociología, Universidad Nacional de General Sarmiento, Argentina.

* Licenciado en Sociología por la UBA y Magíster en Ciencias Sociales con orientación en Educación por la FLACSO. Doctorando UBA. Correo electrónico: diegoab81@gmail.com.

*** Licenciado y Profesor en Sociología por la UBA, Especialista en Demografía Social (UNLu), Magíster en Generación y Análisis de Información Estadística (UNTREF),visitante en la Universidad de California, Berkeley y candidato a Doctor en Ciencias Sociales, UBA. Correo electrónico: nsacco@sociales.uba.ar
} 
herramienta que procura ser útil tanto para la observación de la evolución a lo largo del tiempo como para el análisis de la desigualdad entre diferentes grupos sociales y su relación con el mercado de trabajo.

La aplicación del ISMLU al empleo urbano argentino en el período 2003-2015 permitió distinguir una tendencia positiva entre puntas, pero con tres momentos bien diferenciados: crecimiento importante hasta 2007-explicado principalmente por la baja en la desocupación y el aumento de los ingresos reales-, crecimiento moderado, con oscilaciones entre 2007 y 2011, y valores prácticamente sin modificaciones a partir de entonces. No obstante, el primer semestre de 2015, último dato disponible, presenta el mejor registro de la serie. Al interior de los ocupados, la dimensión ingresos presenta una evolución levemente más positiva que la de la dimensión precariedad, diferencia que se explica por lo sucedido en los primeros años de la serie. Se ha hallado también que la mejor situación relativa que presenta el mercado de trabajo en los años finales del período de estudio es acompañada por una reducción de la desigualdad entre mujeres y varones, entre jóvenes y adultos, y entre los residentes en las regiones socioeconómicamente más y menos favorecidas.

\section{Abstract}

This article presents a multidimensional index that summarizes the different work situations of the economically active population in the urban population of Argentina. To illustrate the application and power of this tool, the Argentine urban labor market dynamics is analyzed during 2003-2015 period.

The Index of Urban Labor Market Situation (IMSLU), was built from the information available of the Permanent Household Survey produced by the National Institute of Statistics and Census. It condensed variables that account for various aspects of the labor market and consists of two large dimensions: informality and income. It is a tool that aims to be useful for both the analysis of the evolution over time and for the analysis of inequality between different social groups and their relationships to the labor market.

The application of the index shows that the Argentine urban employment in the period 2003-2015 experienced a positive trend between points, but with three distinct moments: significant growth until 2007 (mainly due to the decline in unemployment and rising incomes), moderate growth, with 
oscillations between 2007 and 2011 and values virtually unchanged thereafter. However, the first half of 2015, the latest data available, has the best record in the series. The income dimension presents a values slightly more positive than the informality dimension. This difference is explained by what happened in the early years of the series. It has also been found that the best relative situation presented by the labor market in the final years of the period of study was accompanied by a reduction of inequality between women and men, young people and adults and among residents in regions less socioeconomically favored.

\section{Palabras clave}

Calidad del empleo - ingresos - precariedad laboral.

\section{Keywords}

Work quality - income - informality.

\section{Introducción}

Luego de la crisis económica, política y social de 2001-2002, a pesar de la falta de acuerdo sobre la caracterización del período que comenzó en 2003, a la que algunos autores identificaron como la de un nuevo patrón de acumulación (Schorr y Wainer, 2011), los cambios en materia de política económica dieron por resultado un aumento de la actividad y una recomposición general de los indicadores del mercado de trabajo(Damill y Frenkel, 2013; Lindenboim, 2008). Como secuelade la recuperación,se observó un incremento de la demanda agregada de empleo junto a un fortalecimiento del mercado interno(Beccaria, 2008) y un mejoramiento de los indicadores sociales en general, aunque con la permanencia de algunos contrastes en lo que respecta a desigualdad social (Kessler, 2014).

Como resultado de este proceso, el aumento relativo de los ingresos totales de la población ocupada -hasta 2013- mostraba performances diferenciales con "grandes ganadores" -asalariados tanto de servicios como industriales, de establecimientos mayores a cinco ocupados- y "no tan ganadores", trabajadores de establecimientos de pocos ocupados y en general de baja productividad, dando cuenta de la continuidad de un mercado de trabajo heterogéneo en cuanto a sus rasgos estructurales y modos de funcionamiento (Chávez Molina y Sacco, 2015).

Si bien se registró durante el período 2003-2015 una distribución del ingreso más equitativa que en los noventa, este mejoramiento no tuvo como principales destinatarios a quienes se ubicaban en los escalones más bajos de la sociedad, sino 
más bien a sectores de clase media y en particular, de clase media baja (Chávez Molina y Sacco, 2015). Los persistentes niveles de desigualdad social, la continua fragmentación del mercado de trabajo y la creciente diferenciación dentro de la población de menores recursos económicos -potenciados por la persistencia de vastos sectores que aún permanecían sumergidos y fuera del mercado de trabajo- llevó a algunos autores a plantear que la estructura productiva impidió que se logre mejorar la calidad del empleo más allá de un determinado piso y que, además, no se haya avanzado en los últimos años, respecto de los noventa, en cuanto a las posibilidades de movilidad social (Salvia, 2015).

A pesar del conocimiento acumulado sobre los rasgos y características generales de estos procesos, son escasos los estudios que hayan analizado las tendencias del mercado de trabajo a partir de unamirada integral o multidimensional y, a su vez, sintética. Si bien se conocen algunos antecedentes que ya dieron cuenta de la estructura y de la coyuntura del mercado de trabajo en esta etapa en Argentina a partir de un índice complejo (CENDA, 2004; Kostzer, D.; Perrot, B.; Schachtel, L. y Villafañe, S., 2005; CEPED, 2010), no se conocen estudios que partan de las características ocupacionales de cada individuo y que desde su agregación lleguen a resultados finales, lo cual permite no sólo la comparación longitudinal, sino también entre diversos grupos sociales.

El marco de estos debates coyunturales sobre la evolución reciente del mercado de trabajo inspiró la necesidad de proponer una herramienta analítica que condense diferentes aristas relacionadas con el empleo. Por ello, el objetivo de este artículo es presentar la metodología de construcción de un índice resumen (sintético) de calidad del empleo. El índice fue pensado como herramienta complementaria para el análisis de la dinámica del mercado de trabajo, en función de dar cuenta de las diferentes situaciones en las que se encuentran aquellos que conforman la población económicamente activa (PEA). Sobre la base deesta propuesta se realizó un primer análisis para observar los cambios en las tendencias socio-económicas y laborales, de forma global y condensada, durante el período 2003-2015 en Argentina -a nivel del total del país.

Sin dejar de lado la consideración de que la situación del mercado de trabajo se manifiesta en términos de los diferentes atributos de las ocupaciones y como producto de una red compleja de interacciones entre distintas variables económicas (Monza, 1993: 65), en la elaboración del Índice de Situación del Mercado Laboral Urbano (ISMLU) se asumieron como principios ordenadores las disposiciones legales vigentes en materia laboral en el país -que regulan, fundamentalmente, los derechos de los trabajadores en relación de dependencia-, teniendo en cuenta la concepción "moderna" del trabajo y sus condiciones de funcionamiento, desarrollada por el Centro de Estudios e Investigaciones Laborales (Neffa, 1995). 
El trabajo se estructura en tres partes. En primer lugar, en Antecedentes se discuten algunas metodologías sobre medición de calidad de trabajo existentes en la literatura tanto internacional como para el caso de Argentina. En la sección Fuente y Método se describen la fuente -Encuesta Permanente de Hogares-, las variables, la metodología y el marco conceptual utilizado para la construcción del índice. La sección Resultados, a modo de ejemplificar la potencialidad de la herramienta, aborda el análisis del mercado de trabajo urbano argentino en los últimos años, considerando su evolución y sus dos dimensiones -precariedad e ingresos-, y las diferencias entre distintos grupos sociales. Adicionalmente, se plantean conclusiones, fortalezas y debilidades de la herramienta y posibles líneas de trabajo a futuro.

\section{Antecedentes}

En los estudios económicos suele asociarse la calidad del empleo al nivel de los salarios, remuneraciones o retribuciones al trabajo, mientras que en las perspectivas socio-laborales o de las relaciones del trabajo es en general asociado a las condiciones de trabajo. Propuestas recientes en economía y en estudios socio-económicos han formulado enfoques con dimensiones adicionales para la definición de la calidad del empleo.

Desde la teoría del capital humano se reconoce la heterogeneidad tanto de los puestos de trabajo como de los trabajadores, y de esta forma se sugiere diferenciar la calidad del empleo de acuerdo a las calificaciones involucradas en un puesto en particular con respecto a las habilidades de los trabajadores. En un nivel general, las distancias observadas entre estas dos dimensiones podrían ser utilizadas como indicador de la calidad del empleo, considerando las brechas como "fallas" en la participación en la educación o en actividades de formación profesional (Becker, 1983).

Otro abordaje frecuente es aquel que parte de esquemas de prestigio ocupacional o aquellos que "rankean" empleos de acuerdo a los ingresos o los estudios más subjetivos que miden satisfacción en el trabajo, que proponen medidas resumen a partir de estimaciones del "deseo" general de puestos de trabajo específicos a partir de un índice multidimensional de "calidad del trabajo" (Jencks, C.; Perman, L. y Rainwater, L., 1988; Muñoz de Bustillo Llorente y Fernández Macías, 2005; Martel y Dupuis, 2006; Fernández et al., 2009; Royuela et al., 2009; Rovira et al., 2012).

Desde un marco teórico más reciente, "la economía de la felicidad" (Layard, 2005) considera el punto de vista subjetivo de los trabajadores para observar la calidad del empleo, mediante la aplicación de encuestas específicas de satisfacción, mientras que la propuesta sugerida por Green (2006) reconoce el carácter multidimensional de la calidad del empleo al estudiar la evolución de diferentes dimensiones -incluyendo las habilidades, el esfuerzo de trabajo y la intensificación, las características sociales de los trabajadores, los salarios, el riesgo, la inseguridad laboral y el bienestar. 
En Argentina, el Centro de Estudios de Población y Desarrollo (CEPED, 2010)realizó, a partir de las definiciones de trabajo decente elaboradas por la Organización Internacional del Trabajo, un índice de Condiciones de Trabajo teniendo en cuenta la cantidad y calidad de empleos y el nivel de remuneraciones, mientras que el Centro de Estudios para el Desarrollo Argentino (CENDA, 2004) presentó un "Índice Global de Condiciones del Trabajo" que consideraba la cantidad de empleo, la calidad y el salario real. El Ministerio de Trabajo, Empleo y Seguridad Social publicó, a su vez, un Índice de Fragilidad Laboral, teniendo en cuenta tres tipos de déficits -cantidad y calidad de empleo e ingresos- derivados de la conceptualización de vulnerabilidad de Robert Castell (Kostzer et al., 2005).

Es habitual utilizar una gran variedad de indicadores para dar cuenta de los cambios estructurales y coyunturales del mercado de trabajo -tasas de desocupación, subocupación, trabajo no registrado, niveles salariales, etc. Desde esta aproximación suele abordarse una determinada dimensión de la estructura del mercado de trabajo, centrada sobre una parte de la población; por ejemplo, la población económicamente activa subocupada o las características de los ocupados asalariados no registrados.

Aunque la mayoría de los enfoques socio-económicos se basa en las definiciones multidimensionales de la calidad del empleo, persiste un debate sobre la consideración o bien de las varias dimensiones constitutivas del mercado de trabajo, o bien en busca de resumir la calidad del trabajo en una sola variable, la cual sería el nivel de los salarios para la teoría neo-clásica o la satisfacción del empleo de acuerdo a otros marcos conceptuales.

Si bien los indicadores tradicionales sobre la situación del empleo y el trabajo se encuentran interrelacionados entre sí, sus correspondencias no suelen ser estáticas y su elasticidad puede transformarse de acuerdo a otros factores: resulta lógica la correlación entre precariedad laboral o niveles salariales y la tasa de desocupación, pero la intensidad de dicha correlación varía, por ejemplo, de acuerdo al grado de regulación estatal -y de la efectividad de esta regulación.

Con puntos en común con estas propuestas, el índice que se presenta en este artículo se diferencia de aquellas metodologías ya que postula su cálculo según la asignación de puntajes a las características laborales de cada individuo y desde su agregación llega a resultados finales, lo cual permite no sólo la comparación longitudinal, sino también entre diversos grupos -por edad y sexo, educación, lugar de residencia, rama de actividad, etc. Además, toma en cuenta otras variables y, lógicamente, parte de otra estructura de ponderaciones.

\section{Metodología y fuente}

Operacionalmente, el ISMLU se construye sobre la base de la información disponible en las bases de datos -publicada para usuarios externos- de la Encuesta Permanente de Hogares (EPH) del Instituto Nacional de Estadística y Censos (INDEC), en su versión 
continua y para todos los aglomerados urbanos durante el período 2003-2015. De esta forma, el propio marco conceptual de la EPH (Pok, 1992; INDEC, 2011) impregna la perspectiva de análisis adoptada, dado que las definiciones operacionales que conlleva el ISMLU en su búsqueda de jerarquizar las diferentes situaciones no pueden apartarse ni exceder la información -y el tipo de datos- que esta fuente brinda.

Los lineamientos conceptuales generales de la EPH ubican al concepto de precariedad laboral como dimensión confluente en el cambio de la estructura social y las estrategias familiares de vida. Este espacio "se centra en el conjunto de atributos que caracterizan en este nivel a los actores sociales involucrados, hace referencia al ámbito de ocurrencia de los fenómenos vinculados a lo laboral y cuya instancia más institucionalizada es el mercado de trabajo" (Pok, 1992: 5). Este referente conceptual no incorpora de forma directa la dimensión de precariedad, sino que rescata aspectos bajo los cuales se expresan las dimensiones de la estructura social y las estrategias familiares de vida, a saber: la participación en el proceso de trabajo, la división del trabajo y las relaciones sociales de producción y, en este sentido, la precariedad laboral implica realizar cortes a través de esas dimensiones (Pok, 1992).

Las variables e indicadores considerados en la construcción de las dimensiones, así como las categorías y valores que asumen, partieron de las definiciones operacionales y conceptuales utilizadas en la EPH, por lo cual el ISMLU es una herramienta estrictamente ligada a dicho referente empírico. Las variables utilizadas corresponden en todos los casos a distintos aspectos de las características de la situación ocupacional de las personas ${ }^{1}$, indistintamente del sexo, la edad, el nivel educativo, etc., de cada unade ellas.

EI ISMLU considera dos grandes dimensiones referidas a la situación laboral de las personas: precariedad e ingresos. Cabe señalar que el índice construye una medida integral sobre la precariedad del mercado de trabajo, puesto que los ingresos constituyen, lógicamente, un aspecto central en la calidad de una ocupación. Por ello, la dimensión definida aquí como precariedad debe ser entendida en un sentido restringido, apuntando a determinar el grado de estabilidad-vulnerabilidad de la situación ocupacional; como resulta lógico, otras dimensiones sin dudas relevantes para conocer la calidad de los empleos, como la satisfacción "subjetiva", los efectos y riesgos sobre la salud física y mental, etc., no son considerados en el ISMLU, no por una decisión conceptual o metodológica, sino simplemente porque la EPH no indaga sobre estas cuestiones.

Para cada una de las dimensiones se construyó un subíndice, y de su promedio simple surge el valor del ISMLU-es decir, se aplicó un criterio de equiponderación a nivel de

\footnotetext{
${ }^{1}$ Se ha procurado que la construcción de las dimensiones precariedad e ingresos sean totalmente autónomas. Por ello, no se utiliza la información sobre los ingresos para la clasificación de las personas de acuerdo al nivel de precariedad de su ocupación, aun cuando en ciertos casos, particularmente en el de patrones y cuentapropistas, podría considerarse de utilidad.
} 
dimensión. Tanto el índice general como los subíndices varían entre 0 y 100 puntos, donde 0 implica la peor situación y 100, la mejor.

El universo contemplado incluye a la población urbana de 10 y más años económicamente activa, y a una pequeña porción de inactivos, los desocupados "desalentados"-a todo este conjunto se lo denominará "PEA ampliada"(PEAA). A cada una de las personas incluidas en este universo se le asignó un puntaje para cada dimensión y, lógicamente, para el índice general.

La lógica que subyace a la construcción de este índice es la medición de la distancia entre las características ocupacionales de las personas y un umbral, a partir del cual se considera como "satisfactoria" una situación-en función de criterios normativos y de los indicadores que habitualmente se han utilizado para caracterizar la situación del mercado de trabajo. Al igual, por ejemplo, que la metodología de la medición de la pobreza por ingresos, el ISMLU no señala diferencias entre quienes se encuentran por encima del umbral establecido ${ }^{2}$.

\section{Especificidades, ventajas y limitaciones de la propuesta}

En primer lugar, los límites definidos para considerar una situación laboral como satisfactoria son "exigentes" en términos de la situación del mercado laboral, en vistas de la distancia que separa hoy la situación real de los trabajadores de losderechos y las normas que los regulan y protegen, y que son el insumo que buscan reflejar operacionalizar-las ponderaciones asignadas ${ }^{3}$.

Segundo, las ponderaciones asignadas son arbitrarias en tanto que es el juicio de los investigadores el que asigna valor -y con ello, distancia- a las distintas situaciones; cierto es que este es un punto especialmente crítico en la construcción metodológica, pero esta herramienta que aquí se presenta no pretende concluir, sino simplemente impulsar el debate-y adicionalmente, es posible de replicar. Si bien es permisible que la aplicación de herramientas estadísticas más complejas podría ayudar a tomar ciertas

\footnotetext{
2 Tanto un asalariado registrado con un sueldo actual de \$15 mil como un patrón de una mediana empresa con ingresos por $\$ 100$ mil reciben la máxima calificación, puesto que, según las definiciones aquí consideradas, ambas situaciones son satisfactorias, es decir, se ubican por encima del umbral.

${ }^{3}$ Por ejemplo, la Constitución consagra el derecho al trabajo, distintas leyes regulan la obligación de los patrones de inscribir al personal asalariado en la seguridad social y de reconocer sus derechos al aguinaldo, vacaciones, licencia por enfermedad, y también patrones y cuentapropistas deben inscribirse legalmente, como trabajadores autónomos, para desarrollar sus tareas. Como es conocido, una porción importante de la población no trabaja, aunque lo desea, otra es contratada en forma ilegal, etc. En la dimensión ingresos se fija un umbral mínimo equivalente a un $50 \%$ más de lo que la medición estadística de la Canasta Básica Total indica que un hogar tipo necesitaría mínimamente para satisfacer sus necesidades básicas alimentarias y no alimentarias, y este umbral es exigente-término indefectiblemente relativo en última instancia. Otro enfoque podría considerar el umbral en el 50\% de la CBT -es decir, 3 veces menor al aquí planteado- a partir del supuesto que en un hogar tipo ambos cónyuges deberían trabajar y que sólo podría determinarse la existencia de falencias si la suma de los ingresos de ambos no alcanza al valor de la CBT.
} 
decisiones $-\mathrm{y}$, sin dudas, se trata de un ejercicio pendiente-, tampoco se debe perder de vista que un índice construido únicamente con herramientas estadísticas podría ocultar, detrás de la objetividad de los resultados, sinsentidos conceptuales y/o normativos. En última instancia, el problema aquí radica en que el ISMLU justamente juega el rol de variable dependiente, es decir, de aquello que se procura explicar.

En tercer lugar, la asignación de valores numéricos a las distintas situaciones laborales constituye un tema crucial y sobre el cual la decisión ha sido operacionalizar criterios normativos, lo cual se enlaza con una tradición relativamente reciente pero con gran repercusión, ligada a la medición de las condiciones de vida en arreglo a un enfoque de derechos, específicamente para la medición multidimensional de la pobreza (Alkire y Foster; 2007; Alkire y Robles, 2015; Beccaria, 2010; CEPAL, 2014; CEPAL-UNICEF, 2010; CONEVAL, 2012). Este enfoque supone definir umbrales de privación para los indicadores sobre labase detratados internacionales, leyes y regulaciones de distinto orden definiendo diferentes profundidades de privación -umbrales leves, moderados, graves, extremos, etc.-; lógicamente, estas definiciones no se realizan en un espacio vacío, sino partiendo de la base a la tradición de medición de condiciones de vida en cada contexto.

Cuarto, las definiciones conceptuales y metodológicas del IMSLU se inspiran en estos principios. Si bien es un punto sobre el cual se debe profundizar, recurrir a procedimientos estadísticos complejos para establecer objetivamente la estructura de ponderaciones no es per se una solución superadora. Básicamente, porque para realizar este tipo de operaciones es preciso partir de algún tipo de definición de calidad del empleo, lo cual, en un punto, implicaría caer en situaciones tautológicas. En definitiva, para llegar a un escenario ideal, en primer lugar deberían realizarse investigaciones para conocer la opinión de la propia población acerca de cuáles serían los temas relevantes y los umbrales mínimos en relación con la calidad del mercado de trabajo y transformar esas opiniones en una estructura de ponderación; y, en segundo lugar, relevar una encuesta a hogares que además de la información para satisfacer la construcción de los indicadores clásicos del mercado de trabajo, permita contar con la información necesaria para dar cuenta de las dimensiones, indicadores y umbrales definidos como relevantes por la población.

Finalmente, el ISMLU busca dar cuenta de las diferentes situaciones, jerarquizándolas, que no llegan a ser englobadas dentro de lo que aquí se considera satisfactorio-es decir, se busca establecer grises y no limitar la mirada a una simple dicotomía. A continuación, se describe la estructura interna de cada una de las dimensiones.

\section{Dimensión precariedad}

Esta dimensión da cuenta, en sentido amplio, de la calidad de la ocupación, en términos de su formalidad y estabilidad. Además, aquí se clasifica tanto a los ocupados como a los desocupados con ocupación en los seis meses anteriores a ser encuestados, 
en tanto que el resto de los desocupados reciben, por definición, 0 puntos ${ }^{4}$. Como se desarrollaa continuación, en ciertos casos la falta de información directa conlleva la necesidad de utilizar variables proxy de lo que esta dimensión busca reflejar. La intensidad de la ocupación, así como el tiempo transcurrido desde el último trabajo o changa, en el caso de los desocupados, entra en juego a modo de penalización o quita de puntos ${ }^{5}$.

\section{Ocupados}

En todos los casos se consideraron las características de la ocupación principal. La primera división corresponde a las categoría ocupacional: dentro de cada una de ellas la asignación de puntaje se realiza siguiendo criterios diferentes ${ }^{6}$.

Asalariados: el puntaje otorgado varía de acuerdo al grado de formalidad de la relación, oscilando entre 0 puntos para quienes no tienen ningún derecho laboral y 100 puntos para quienes disponen de todos ellos. A partir de la información disponible, se consideran dos subdimensiones:

Aportes al régimen previsional (60 puntos), de acuerdo a tres situaciones7:

- Con descuento jubilatorio: 60 puntos.

- Sin descuentos jubilatorios, pero con aportes propios: 30 puntos.

- Sin descuentos ni aportes propios: 0 punto.

Otros derechos sociales (40 puntos), de acuerdo a cuatro indicadores: vacaciones pagas, aguinaldo, días pagos por enfermedad y obra social. Cada uno de ellos otorga 10 puntos a quien dispone de él y 0 punto a quien no $^{8}$.

\footnotetext{
${ }^{4}$ Esta distinción al interior de los desocupados procura una aproximación a distinguir entre situaciones donde prevalece el desempleo coyuntural, de corta duración -y, por tanto, con mayor probabilidad de rápido retorno a ocupar un puesto de trabajo, y donde lo que prevalece es el desempleo crónico.

${ }^{5}$ La construcción de esta dimensión recoge los aportes de distintos autores que han trabajado sobre la precariedad (Pok, 1992; Groisman et al.).

${ }^{6}$ La utilización de diferentes variables según la categoría ocupacional obedece a la falta de información uniforme para todas ellas.

${ }^{7}$ La distinción entre quienes reciben descuentos jubilatorios y quienes aportan por su cuenta obedece a que en el primero de los casos el descuento es una clara señal de la presencia de un empleado legalmente registrado, mientras que el aporte propio, cuando no es acompañado por el descuento obligatorio, da cuenta de una situación de fragilidad en tanto que la relación de dependencia fáctica es encubierta, por ejemplo, bajo una forma legal de contratación de servicios brindados por un trabajador independiente.

${ }^{8} \mathrm{Si}$ bien todos aquellos asalariados a los que se le realizan descuentos jubilatorios deberían estar sujetos al disfrute de esos derechos, su enumeración por separado apunta fundamentalmente a establecer diferencias entre aquellos asalariados a los que no se les realizan dichos descuentos. Así, por ejemplo, un trabajador sin descuento ni aportes jubilatorios propios que no dispone de ninguno de estos cuatro beneficios recibirá 0 puntos, mientras que otro en la misma situación previsional a quien se le reconocen vacaciones pagas, días por enfermedad y aguinaldo obtendrá 30 puntos.
} 
Los asalariados cuya calificación no es profesional -es decir, es técnica, operativa o no calificada- reciben una penalización o quita del $50 \%$ de los puntos en los casos en que su ocupación no corresponda a un empleo sin tiempo de finalización ${ }^{9}$.

Cuentapropistas $^{10}$ : los cuentapropistas con calificación ocupacional profesional o técnica reciben 100 puntos.En contrapartida, a quienes se desempeñan en ocupaciones no calificadas se les otorga 0 punto.Es entre los cuentapropistas operativos donde radica la mayor complejidad de la clasificación propuesta. Aquí se optó por realizar la asignación de puntaje de acuerdo a la disponibilidad de bienes con los que cuentan para el desarrollo de su actividad:

100 puntos: con local o establecimiento propio,con local alquilado/prestado y con vehículo o con maquinarias propias.

75 puntos: sin local y con vehículo propio.

50 puntos: con local alquilado/prestado y sin vehículo y sin maquinarias propias,sin local y con vehículo alquilado/prestado y con herramientas propias.

25 puntos: sin local y con vehículo alquilado/prestado y sin herramientas propias, sin local y sin vehículo y con herramientas propias.

0 punto: sin local, sin vehículo y sin herramientas propias.

\begin{abstract}
${ }^{9}$ Los trabajos con tiempo de finalización incluyen changas, trabajos transitorios, por tarea u obra, suplencias, períodos de pruebas, becas o pasantías, planes de empleo, etc. Cabe señalar que la decisión de no utilizar la variable que identifica si la ocupación principal corresponde a un plan de empleo obedece a que esta información fue discontinuada a partir de la base de microdatos del primer trimestre de 2011. Los empleados de servicio doméstico no reciben esta penalización ya que la información sobre tiempo de finalización no es relevada por la EPH. Debido a las características intrínsecas de esta actividad, se ha decidido no contemplar un proxy para esta penalización. En el caso de los profesionales, la no aplicación de esta penalización obedece al supuesto de que la inestabilidad dentro de esta calificación ocupacional no es intrínsecamente negativa, puesto que quienes desarrollan este tipo de tareas tienen altas probabilidades de estar en condiciones de "elegir" tal situación. El mismo supuesto rige en las penalizaciones dispuestas para las restantes categorías ocupacionales.
\end{abstract}

\footnotetext{
${ }^{10} \mathrm{En}$ el caso de los trabajadores independientes, especialmente en el de los cuentapropistas, es habitual encontrar la utilización del nivel educativo como proxy de la precariedad de su situación laboral, ante la escasa y a la vez compleja información existente para su clasificación. En este caso, se ha optado por no utilizar el nivel educativo sobre la base de dos razones: la primera obedece al criterio de no utilizar variables exógenas a la caracterización de la situación laboral; la segunda razón se basa en la necesidad de evitar trasladar la posesión de capital educativo a la caracterización laboral. Así, en un contexto de alta desocupación, la probabilidad de que haya cuentapropistas con alto nivel educativo en tareas de baja calificación es mayor que en contextos más favorables: si se aplicara el nivel educativo para caracterizar a un cuentapropista, su clasificación sería inelástica, es decir, ajena a los vaivenes del mercado laboral y, por tanto, iría en contra del objetivo perseguido por el ISMLU. Tampoco se ha utilizado, en ningún caso, la información referida a la rama de actividad, dado que resulta discutible la jerarquización de las actividades sin información adicional; en caso de recurrir a dicha información, se estaría violentando el principio de "autonomía" de las variables utilizadas: por ejemplo, si se establece un ranking de ramas de actividad de acuerdo al ingreso medio de los empleados en cada una de ellas, la información sobre los ingresos estaría influyendo tanto en la dimensión precariedad como en la dimensión de ingresos propiamente dicha. Una razón adicional para la no utilización del nivel educativo y la rama de actividad en la construcción del ISMLU es que en ambos casos se trata de variables independientes de sumo interés para realizar comparaciones a partir del mismo.
} 
Adicionalmente, los cuentapropistas, a excepción de los profesionales, reciben una penalización o quita de puntos del $50 \%$ en los casos en que la actividad en que se desempeñan se haya iniciado dentro del lapso de los últimos 6 meses.

Patrones: todos los patrones con calificación ocupacional profesional reciben 100 puntos.Entre los patrones con calificación técnica ${ }^{11}$ se distingue a quienes se desempeñan en establecimientos donde trabajan cuatro o más personas -bajo el supuesto que tienen asu mando a tres o más empleados ${ }^{12}{ }_{-}$, quienes reciben 100 puntos, y a quienes se desempeñan en establecimientos de hasta 3 personas -es decir, con uno o dos empleados-, casos en los que la asignación del puntaje está ligada a la disponibilidad de bienes:

100 puntos: con local o establecimiento propio,con local alquilado/prestado y con vehículo o con maquinarias propias,sin local y con vehículo propio.

80 puntos: con local alquilado/prestado y sin vehículo y sin maquinarias propias,sin local y con vehículo alquilado/prestado y con herramientas propias.

60 puntos: sin local y con vehículo alquilado/prestado y sin herramientas propias,sin local y sin vehículo y con herramientas propias.

50 puntos: $\sin$ local, sin vehículo y sin herramientas propias.

Los patrones con calificación técnica reciben una penalización o quita de puntos del $50 \%$ en los casos en que su actividad se haya iniciado dentro del lapso de los últimos 6 meses.

Trabajadores familiares sin remuneración fija: en todos los casos reciben 0 puntos. Independientemente de la categoría ocupacional y los puntos obtenidos hasta aquí, todos los ocupados que son subocupados demandantes ${ }^{13}$ reciben una penalización del $50 \%^{14}$.

\footnotetext{
${ }^{11}$ La calificación ocupacional de los patrones, por definición, es profesional o técnica. Por esta razón, a diferencia de los cuentapropistas, que reciben el máximo puntaje cuando desempeñan tareas técnicas, en el caso de los patrones técnicos pueden ser calificados con un menor valor de acuerdo a la cantidad de empleados y al capital disponible.
}

\footnotetext{
${ }^{12}$ Se asume aquí que la cantidad de empleados que depende de un patrón es igual al tamaño del establecimiento menos uno, justamente, el patrón. Sin embargo, cabe señalar que en los casos en que el patrón comparta su situación con uno o más socios, la cantidad de empleados es sobreestimada con la utilización de este supuesto.

${ }^{13}$ En el caso de los ocupados que no trabajaron en la semana de referencia, debido a la falta de información se ha tomado la siguiente decisión: aplicar la penalidad en aquellos casos en que la persona haya declarado que la semanapasada quería trabajar más horas, que estaba dispuesto a hacerlo y que estuvo buscando otro empleo, ocupación o actividad: en este caso se asumió que se trata de un subocupado demandante.

${ }^{14}$ Un punto que requiere justificación es la no penalización para subocupados no demandantes y sobreocupados.

En el primer caso, se asume que la no demanda de trabajar mayor cantidad de horas refleja la "elección" de la decisión de no hacerlo, es decir, estaría expresando su conformidad con la situación.
} 


\section{Desocupados con última ocupación en los últimos 6 meses}

La clasificación de este grupo se realiza considerando las características de la última ocupación ${ }^{15}$. Al igual que en el caso de los ocupados, la primera distinción que se realiza aquí es de acuerdo a la categoría ocupacional, en este caso, correspondiente a la última ocupación.

Asalariados: respecto a la información sobre la vigencia de los derechos que corresponden a una relación de dependencia acorde a los parámetros de la ley laboral, sólo se cuenta con la distinción entre quienes recibían y quienes no descuentos jubilatorios en su última ocupación. Con esta información y la referida a la calificación ocupacional, se procede a la puntuación de quienes integran este grupo:

Con descuento jubilatorio, independientemente de la calificación ocupacional: 100 puntos.

Profesional o técnico sin descuento jubilatorio: 50 puntos.

Operativo sin descuento jubilatorio: 25 puntos.

No calificado sin descuento jubilatorio: 0 puntos.

Como en el caso de los ocupados, los desocupados que en su última ocupación se desempeñaron como asalariados no profesionales reciben una penalización del $50 \%$ cuando la misma no se correspondía con un empleo sin tiempo de finalización.

Cuentapropistas: la escasa información disponible ${ }^{16} \mid l$ eva a clasificar a los desocupados ex cuentapropistas únicamente a partir de la calificación de la última ocupación:

Profesionales y técnicos: 100 puntos.

Operativos: 50 puntos.

No calificados: 0 puntos.

A excepción de quienes se desempeñaron por última vez en un empleo de calificación profesional, los cuentapropistas cuya última actividad había comenzado menos de un año antes de su finalización reciben una penalización del $50 \%$.

En el caso de los sobreocupados existe una gran variedad de situaciones de diversa índole que la explican (por ejemplo, una empleada doméstica con "cama adentro" y 12 horas de servicio diario de lunes a sábado, frente a un patrón de una mediana empresa que dedica 10 horas diarias a su actividad). Se asume aquí que las variables involucradas en la determinación del nivel de precariedad son suficientes para determinar si se trata o no de una "elección". Además, la dimensión ingresos, al considerar el ingreso horario y no el ingreso total, también penalizará de hecho a las ocupaciones que insumen gran cantidad de horas y son retribuidas con ingresos escasos.

${ }^{15} \mathrm{Si}$ bien se ha procurado mantener en la mayor medida posible la correspondencia entre la clasificación de este grupo y los criterios utilizados en el caso de los ocupados, la información disponible en la batería de preguntas de la EPH para unos y otros torna inevitables los cambios en los parámetros a considerar.

${ }^{16}$ La batería utilizada por la EPH para caracterizar la última ocupación de los desocupados no contiene preguntas sobre la disponibilidad de capital. 
Patrones: los ex patrones de calificación profesional reciben 100 puntos. A quienes se desempeñaban en ocupaciones técnicas se adjudican 100 puntos en los casos que el tamaño del establecimiento sea mayor a tres trabajadores y 75 puntos cuando no supere ese valor. También aquí los técnicos se penalizan con una quita del $50 \%$ de los puntos cuando el inicio de su última actividad haya sido menos de un año antes de su finalización. A los puntos obtenidos hasta aquí por los desocupados que perdieron en forma reciente su empleo, se le aplican penalizaciones de acuerdo al tiempo transcurrido hasta la última actividad ${ }^{17}$ :

$70 \%$ de quita a quienes tuvieron un trabajo o changa en el último mes.

$80 \%$ a quienes trabajaron por última vez entre uno y tres meses atrás.

$90 \%$ a quienes hayan finalizado su última ocupación más de tres y hasta seis meses atrás.

Trabajadores familiares sin remuneración fija: en todos los casos reciben 0 punto.

\section{Desocupados sin ocupación en los últimos seis meses}

Se incluyeron aquí los desocupados con ocupación anterior que no trabajaron en los seis meses anteriores -independientemente de las características de su última ocupación-, a los desocupados sin ocupación anterior y a los desocupados desalentados: por definición, para este grupo el puntaje otorgado es 0 .

\section{Dimensión ingresos}

Aquí se contempló el ingreso horario de cada ocupado, considerando las horas trabajadas y los ingresos laborales -en ambos casos, tanto de su ocupación principal como de sus ocupaciones secundarias. Todos los desocupados reciben 0 punto.

El puntaje otorgado varía de acuerdo a una escala proporcional que toma como umbral mínimo la no percepción de ingresos en el mes de referencia ( 0 punto) y como umbral máximo un ingreso horario necesario para adquirir 1,5 canastas básicas totales $(C B T)^{18}$ para un hogar tipo ${ }^{19}$-quienes con su ingreso horario alcancen o superen este umbral, reciben 100 puntos $^{20}$.

\footnotetext{
${ }^{17}$ El supuesto sobre el que se asienta esta penalización es que, a mayor tiempo transcurrido desde la última ocupación, menor es la probabilidad de estar ocupado en el corto plazo y menor es la "influencia" que las características de la última ocupación tendrán sobre las características de una ocupación futura. La lógica de esta penalización marca que un desocupado cuya última ocupación cumplía con los requisitos para que la misma sea calificada como satisfactoria y que dejó de trabajar hace menos de un mes puede recibir un máximo de 30 puntos, mientras que si esta ocupación finalizó entre uno y tres meses atrás el techo se reduce a 20 puntos y a 10 en caso que su finalización haya ocurrido entre más de tres y seis meses. Cabe señalar que, por esta misma razón, los desocupados que hace 6 meses o más no trabajan reciben 0 puntos.

${ }^{18}$ Consideramos esta forma más adecuada para definir un umbral que, por ejemplo, el Salario Mínimo, Vital y Móvil (SMVM), debido a que éste responde a una definición normativa y no "real". Previo a la devaluación de 2001, el SMVM se mantuvo durante muchos años (desde 1993) en \$200, en un contexto de estabilidad de precios. A inicios de 2015, el SMVM se ubicaba en $\$ 6.060$, que en términos reales más que
} 
Para quienes perciban ingresos horarios inferiores al límite máximo establecido, el puntaje se otorga de acuerdo a la siguiente fórmula:

$$
\text { YHLInd/IHRef } 100
$$

Donde,

YHRef (Ingreso Horario de Referencia): YHRef = 1,5 CBT_Ht / HMNl, donde $\mathrm{CBT}_{-} \mathrm{H} \mathrm{t}=$ Valor mensual de la CBT para un hogar "tipo" (3,09 Adulto Equivalente).

$$
\mathrm{HMNI}=\text { Horas mensuales "normales" (45hs semanales * 4,3 semanas). }
$$

\title{
YHLInd (Ingreso Horario Laboral): YTI_L /HMTr, donde \\ YTI_L= Ingreso laboral total del mes de referencia. \\ $\mathrm{HMTr}=$ Horas mensuales trabajadas (horas semanales * 4,3 semanas) ${ }^{21}$.
}

Como puede observarse, no se trata de una medida relativa, sino absoluta, es decir, no considera las diferencias entre los ingresos de las personas, sino el porcentaje que representan estos ingresos respecto de un umbral definido: cuando los ingresos percibidos sean mayores a dicho umbral, indistintamente de por cuanto lo sean, se otorga el máximo puntaje (100 puntos) ${ }^{22}$.

duplicaba el valor de la década del 90. Tampoco consideramos conveniente utilizar el salario promedio, puesto que resultaría tautológico en relación con los fines perseguidos por el ISMLU.

\footnotetext{
${ }^{19} \mathrm{El}$ "Hogar tipo" considerado corresponde al Hogar $\mathrm{N}^{\circ} 2$ definido por el INDEC en sus comunicados de prensa y está conformado por cuatro miembros: un jefe varón de 35 años, su esposa de 31 años, un hijo de 5 y una hija de 8 años. El jefe equivale a 1,00 adulto equivalente, la esposa a 0,74 adulto equivalente, el hijo a 0,63 y la hija a 0,72 de adulto equivalente. La suma total del hogar son 3,09 adultos equivalentes. Para detalles sobre la metodología de estimación de la CBT y la línea de pobreza, véase INDEC, 2003.
}

\begin{abstract}
${ }^{20}$ Desde principios de 2007 las estadísticas de precios del INDEC no resultan fiables. Por tal razón, el valor de la CBT utilizado aquí se ha calculado a partir de diversas fuentes: desde 2003 a 2006, CBT-INDEC; desde 2007 a 2010 se ha aplicado al valor de la CBT-INDEC de diciembre de 2006, la evolución de la CBT-Paraná elaborada por la Dirección Provincial de Estadística de la Provincia de Entre Ríos (cuya publicación fue discontinuada a fines de 2010). Desde 2011 se ha considerado la variación de la Canasta Total del Hogar 1 (matrimonio compuesto por una mujer y un varón, ambos de 35 años, activos, con dos hijos varones de 6 y 9 años y propietarios de la vivienda) del Sistema de Canastas de Consumo del Gobierno de la CABA (dado que hasta enero de 2013 sólo se informó el valor para noviembre, las variaciones mensuales al interior de cadaaño fueron estimadas sobre la base de la evolución del "IPC Congreso").Cabe señalar que los valores de la CBT corresponden a cada uno de los meses, mientras que en las bases trimestrales no es posible conocer en qué mes fue encuestado el hogar $y$, por tanto, el período de referencia para realizar la comparación exacta; por tal motivo se ha utilizado el promedio de los tres meses de referencia correspondientes a cada uno de los trimestres. Finalmente, los valores de la CBT han sido ajustados según los coeficientes regionales (INDEC, 2002).
\end{abstract}

\footnotetext{
${ }^{21}$ En el caso de los trabajadores que no trabajaron en la semana de referencia, dado que no es posible estimar las horas trabajadas mensuales, se sigue la misma lógica, pero confrontando en forma directa los ingresos laborales totales con el valor de 1,5 CBT_Ht.

${ }^{22}$ Así, en el segundo trimestre de 2015, un ocupado del Gran Buenos Aires que trabajaba 45 horas semanales y percibía ingresos por $\$ 9.618 .11$ (equivalente a $1,5 \mathrm{CBT}+\mathrm{Ht}$ ) recibe los 100 puntos
} 
Para brindar una idea precisa de cuánto implica el umbral definido y su variación en el tiempo, cabe decir que el valor de la CBT_Ht del tercer trimestre de 2003 era de $\$ 701$, por lo que 1,5 CBT_Ht equivalían a $\$ 1.052$. Este monto dividido por la cantidad de horas consideradas $\left(45^{*} 4,3=193,5\right)$ arrojaba un valor para el YHRef de $\$ 5,43$. En el cuarto trimestre de 2006, el valor de la CBT_Ht era de $\$ 870$ y del YHRef, de $\$ 6,74$. Tres años después, en el último trimestre de 2009 , estos valores alcanzaban a $\$ 1.613$ y $\$ 12,51$, respectivamente. Para el último período analizado en este artículo, el segundo trimestre de 2015 , el valor de la CBT_Ht era de $\$ 6.412$ y el YHRef, de $\$ 49,71$-en todos los casos los valores de los ejemplos corresponden al aglomerado Gran Buenos Aires.

Para marzo de 2016 y con la metodología de valorización descripta, la CBT asciende a $\$ 8.440-1,5$ CBT representan entonces $\$ 12.661$ - y el YHRef, a $\$ 65,43$. Nótese que si el valor horario se hubiese construido siguiendo estas pautas, pero tomando como referencia al salario mínimo, vital y móvil bruto (\$6.060) -recuérdese que la EPH releva ingresos netos-, el umbral habría sido de $\$ 31,38$, es decir, menos de la mitad del aquí utilizado; si en cambio se hubiese considerado el valor bruto y desestacionalizado de la mediana del salario del sector privado registrado ( $\$ 13.748$ en marzo de 2016), el ingreso horario de referencia hubiese alcanzado $\$ 71,05$, un valor apenas mayor al considerado-para más detalles ver Tabla 2 en el Anexo.

\section{Índice General}

El Índice General o ISMLU se calcula para cada una de las personas que conforman la PEA ampliada, a partir del promedio simple de las dos dimensiones consideradas.El agrupamiento de los individuos de acuerdo a distintas variables -corte longitudinal, por año o por año y trimestre, pero también por características de la población: sexo, edad, nivel educativo, región de residencia, tipo de hogar- permite obtener un índice resumen de la situación del mercado de trabajo para un determinado conjunto de la población y realizar a partir del mismo comparaciones tanto sincrónicas -respecto a otros grupos- como diacrónicas, es decir, a lo largo del tiempo. Además del ISMLU, es posible analizar la situación para cada dimensión, a partir de los subíndices correspondientes. Tanto el ISMLU como los subíndices de cada una de las dimensiones pueden ser utilizados considerando al total de la PEA ampliada o sólo a los ocupados, evitando así el impacto de las variaciones en el nivel de la desocupación sobre las condiciones laborales de los ocupados en el índice general.

De acuerdo a las definiciones adoptadas, el ISMLU asumiría el valor máximo (100 puntos) si todas las personas que quisieran trabajar estuvieran trabajando, sin desear, aquellos que trabajen menos de 35 horas semanales, hacerlo por una mayor cantidad de horas; si fuesen asalariados y dispusieran de todos los derechos laborales señalados 
por las leyes o bien si fuesen cuentapropistas en ocupaciones técnicas o profesionales u operativas con capital propio, o bien si fuesen patrones profesionales o patrones técnicos de medianas o grandes empresas o de pequeñas empresas con capital propio; y si, en cualquier caso, recibieran ingresos horarios equivalentes o superiores a los necesarios para afrontar el costo de 1,5 CBT_Ht trabajando 45 horas semanales.

Finalmente, a modo de ejemplo, la Ilustración 1 muestra el puntaje en cada dimensión y en el índice general que correspondería a personas en diferentes situaciones laborales, así como los puntajes que asumirían para el grupo. En la dimensión ingresos, se ha considerado un YHRef $=\$ 10$.

\section{Ilustración 1. Ejemplo de puntajes del ISMLU}

\begin{tabular}{|c|c|c|c|}
\hline \multirow{2}{*}{$\begin{array}{l}\text { Ejemplo de situación laboral } \\
\qquad(Y H R e f=\$ 10)\end{array}$} & \multicolumn{2}{|c|}{ Dimensiones } & \multirow{2}{*}{$\begin{array}{c}\text { Índice General } \\
\text { (ISMLU) }\end{array}$} \\
\hline & Precariedad & Ingreso & \\
\hline Desocupado cuya última ocupación finalizó hace un año & 0.0 & 0.0 & 0.0 \\
\hline $\begin{array}{l}\text { Desocupado cuya última ocupación (como asalariado no calificado con } \\
\text { descuentos jubilatorios) finalizó dos meses atrás }\end{array}$ & 20.0 & 0.0 & 10.0 \\
\hline $\begin{array}{l}\text { Asalariado subocupado no demandante sin descuentos ni aportes } \\
\text { jubilatorios, pero con vacaciones y días por enfermedad pagos, en un } \\
\text { empleo sin plazo de finalización con ingresos horarios de } \$ 2\end{array}$ & 20.0 & 20.0 & 20.0 \\
\hline $\begin{array}{l}\text { Asalariado pleno sin ningún derecho laboral con ingresos horarios de } \\
\$ 8,25\end{array}$ & 0.0 & 82.5 & 41.3 \\
\hline $\begin{array}{l}\text { Cuentapropista ocupado pleno con calificación ocupacional técnica y } \\
\text { sin ingresos en el mes de referencia, que inició su actividad hace dos } \\
\text { años }\end{array}$ & 100 & 0 & 50.0 \\
\hline $\begin{array}{l}\text { Asalariado pleno que realiza sus propios aportes jubilatorios y tiene } \\
\text { sólo vacaciones y días por enfermedad pagos en un empleo con tiempo } \\
\text { de finalización eingresos horarios de } \$ 7,5\end{array}$ & 25.0 & 75.0 & 50.0 \\
\hline $\begin{array}{l}\text { Asalariado subocupado demandante con todos los derechos laborales } \\
\text { (sin plazo) e ingreso horario de } \$ 18\end{array}$ & 50.0 & 100.0 & 75.0 \\
\hline $\begin{array}{l}\text { Patrón técnico sobreocupado con } 3 \text { empleados en una actividad iniciada } \\
\text { hace siete meses, con ingresos horarios de } \$ 11\end{array}$ & 100.0 & 100.0 & 100.0 \\
\hline $\begin{array}{l}\text { Asalariado pleno con todos los derechos laborales (en un empleo sin } \\
\text { plazo) e ingreso horario de } \$ 13\end{array}$ & 100.0 & 100.0 & 100.0 \\
\hline Promedio del grupo (PEA Ampliada) & 46.1 & 53.1 & 49.6 \\
\hline Promedio del grupo (ocupados) & 56.4 & 68.2 & 62.3 \\
\hline
\end{tabular}

\section{Resultados}

En esta sección se realiza un análisis del mercado de trabajo en la Argentina para dar cuenta de la potencialidad analítica del ISMLU, en especial en aquellas dimensiones que mayormente suelen utilizarse en estudios sociales. Para ello, primero se abordaron las tendencias generales del índice a lo largo del lapso 2003-2015, luego se observó su comportamiento frente a otras variables económicas, pasando de seguido a un mayor detalle de la variación entre sus dimensiones; finalmente se observaron las diferencias desagregadas por sexo, edad y lugar de residencia. 


\section{ISMLU general según dimensiones}

Al partirde un contexto socioeconómico crítico al comienzo del período de observación, el nivel del ISMLU muestra una mejora notable entre 2003 y 2015. Como se observa en el Gráfico 1 , en el $3^{\circ}$ Trimestre $\left(3^{\circ} \mathrm{T}\right)$ de 2003 el ISMLU para la PEA Ampliada (ISMLU_PEAA) alcanza 43,8 puntos, mientras que en el $2^{\circ} \mathrm{T}$ de 2015 -último período para el que se cuenta con información- este valor se incrementa hasta 64,8 puntos: la mejora fue de 21,1 puntos del índice, alcanzando en términos relativos un $48,2 \%$.

Esta mejora entre puntas oscurece diferencias entre períodos claramente distintos. Entre 2003 y 2007 se observa un crecimiento franco, mientras que a partir de allí los valores muestran una leve tendencia alcista hasta 2011, momento a partir del cual, con leves oscilaciones, los valores se mantienen en niveles similares. En síntesis, poco más de dos terceras partes del crecimiento a lo largo del período considerado del ISMLU_PEAA y del ISMLU_Ocupados se focalizan entre 2003 y 2007, mientras que el período 2011-2015 sólo se registra, entre puntas, el 7,5\% del crecimiento.

Gráfico 1. ISMLU_PEAA, ISMLU_Ocupados e Índices de Precariedad e Ingresos para ocupados por trimestre. Grandes aglomerados urbanos, $3^{\circ} \mathrm{T}$ de 2003 a $2^{\circ} \mathrm{T}$ de 2015

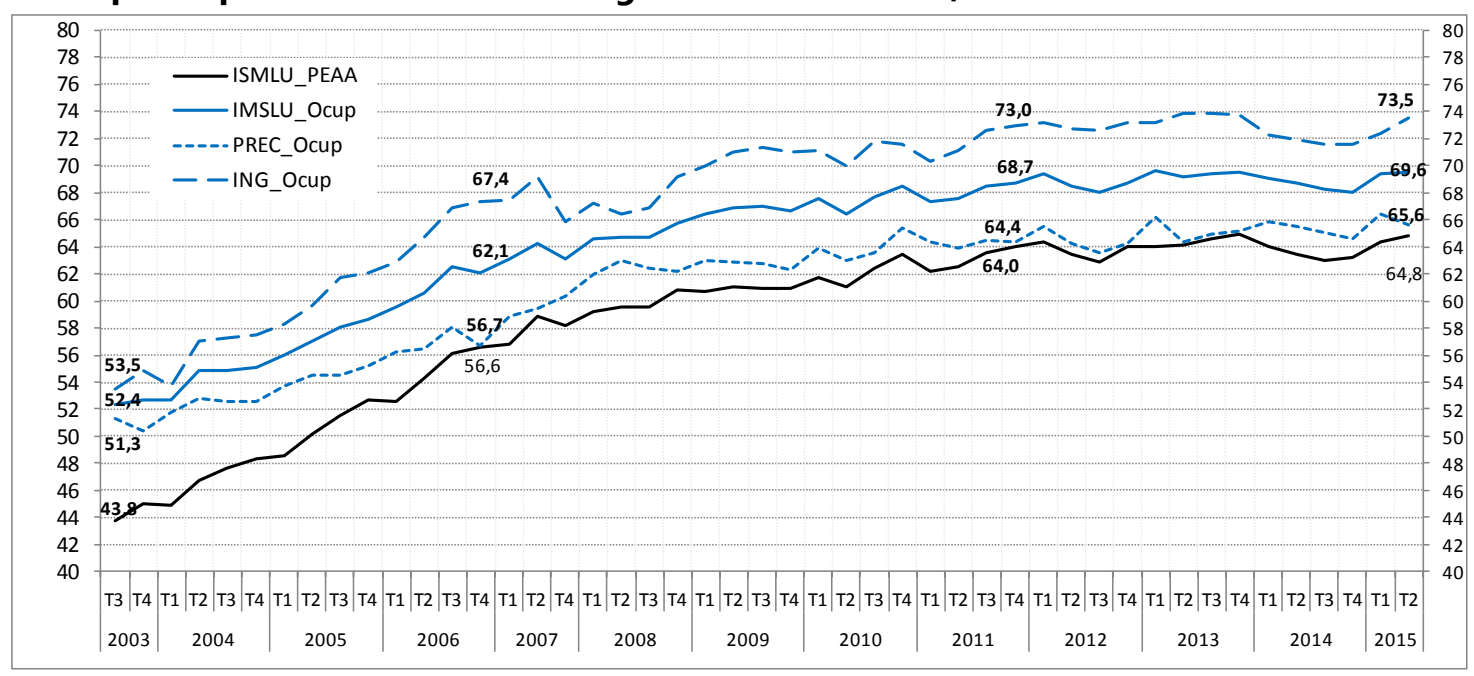

Fuente: bases trimestrales de microdatos de la EPH-Continua (INDEC), $3^{\circ} \mathrm{T}$ de 2003 a $2^{\circ} \mathrm{T}$ de 2015.

En los primeros años de la serie, hasta 2007, el crecimiento del IMSLU_PEAA mostró una mayor intensidad en su crecimiento (30,6\% acumulado) que el ISMLU_Ocupados (20,9\%) -Tabla 1. En cambio, entre 2007 y 2015, el crecimiento relativo, desacelerado sobre todo a partir de 2012, fue similar en ambos índices, alcanzando acumulados de $11,4 \%$ y $9,4 \%$, respectivamente.

Dentro del universo de los ocupados, la variación del índice general y de los subíndices presenta algunas particularidades. Entre 2003 y 2006, la dimensión ingresos explica cerca de dos tercios del crecimiento del ISMLU_Ocupados, que se expandió en 
torno al 5\% interanual. En 2007 y 2008, el crecimiento interanual se ubica en alrededor del 3\%: allí el incremento obedece fundamentalmente a la mejora de la dimensión precariedad -que se ubica en un ritmo interanual apenas inferior al $5 \%$, el más alto de toda la serie- en tanto que el crecimiento en ingresos se reduce del 8,3\% registrado entre 2006 y 2005 al 2,1\% entre 2007 y 2006, y a sólo el 0,8\% entre 2008 y 2007.

Los años siguientes mostrarán tendencias cambiantes-y con varios cambios interanuales no estadísticamente significativos: en 2009, 2011, 2012 y 2015, la mejora en ingresos es levemente superior a la registrada en precariedad, mientras que en 2010 y 2013 ocurre lo contrario. Estas diferencias registradas en la evolución del ISMLU entre los subperíodos 2004-2007, con mejoras sostenidas; 2008- 2011, con desaceleración en el crecimiento y un ritmo oscilante, y 2012-2015, con valores estancados y también oscilantes, se explica en parte debido al fuerte descenso de la tasa de desocupación durante los primeros años que implicó, lógicamente, la reducción de la participación de los desocupados en la PEA Ampliada -aquí también radica gran parte de la explicación sobre la disminución de la brecha entre los valores del ISMLU_PEAA y el ISMLU_Ocupados.

Tabla 1. ISMLU_PEAA, ISMLU_Ocupados e Índices de Precariedad e Ingresos para ocupados por año. Grandes aglomerados urbanos, 2003-2015

\begin{tabular}{|c|c|c|c|c|c|c|c|c|c|c|c|c|}
\hline \multirow{3}{*}{ Año } & \multicolumn{3}{|c|}{ PEA AM PLIADA } & \multicolumn{9}{|c|}{ OCUPADOS } \\
\hline & \multirow[b]{2}{*}{$\begin{array}{l}\text { Puntos } \\
\text { Indice }\end{array}$} & \multicolumn{2}{|l|}{ ISMLU } & \multicolumn{3}{|c|}{ ISMLU } & \multicolumn{3}{|c|}{ PRECARIEDAD } & \multirow[b]{2}{*}{$\begin{array}{l}\text { Puntos } \\
\text { Indice }\end{array}$} & \multicolumn{2}{|c|}{ INGRESOS } \\
\hline & & $\begin{array}{c}\text { Var. } \\
\text { Interanual }\end{array}$ & $\begin{array}{c}100= \\
\text { 2oS } 2003\end{array}$ & $\begin{array}{l}\text { Puntos } \\
\text { Indice }\end{array}$ & $\begin{array}{c}\text { Var. } \\
\text { Interanual }\end{array}$ & $\begin{array}{c}100= \\
\text { 2oS } 2003\end{array}$ & $\begin{array}{l}\text { Puntos } \\
\text { Indice }\end{array}$ & $\begin{array}{c}\text { Var. } \\
\text { Interanual }\end{array}$ & $\begin{array}{c}100= \\
2 \text { o } 2003\end{array}$ & & $\begin{array}{c}\text { Var. } \\
\text { Interanual }\end{array}$ & $\begin{array}{c}100= \\
2 \stackrel{\circ}{\circ} 2003\end{array}$ \\
\hline $2003\left(2^{\circ} \mathrm{S}\right)$ & 44,4 & -- & 100,0 & 52,5 & -- & 100,0 & 50,9 & -- & 100,0 & 54,2 & -- & 100,0 \\
\hline 2004 & 46,9 & -- & 105,7 & 54,4 & -- & 103,5 & 52,4 & -- & 103,0 & 56,4 & -- & 104,1 \\
\hline $2004\left(2^{\circ} \mathrm{S}\right)$ & 48,0 & $8,1 \%$ & 108,1 & 55,0 & $4,6 \%$ & 104,6 & 52,6 & $3,3 \%$ & 103,3 & 57,4 & $5,9 \%$ & 105,9 \\
\hline 2005 & 50,8 & $8,2 \%$ & 114,4 & $\mathbf{5 7 , 5}$ & $5,7 \%$ & 109,4 & 54,5 & $4,0 \%$ & 107,1 & 60,5 & $7,2 \%$ & 111,6 \\
\hline 2006 & 54,9 & $8,2 \%$ & 123,8 & 61,2 & $6,5 \%$ & 116,5 & 56,9 & $4,4 \%$ & 111,8 & 65,5 & $8,3 \%$ & 120,9 \\
\hline 2007 & 58,0 & $5,6 \%$ & 130,6 & 63,5 & $3,8 \%$ & 120,9 & 59,5 & $4,7 \%$ & 117,0 & 67,5 & $3,0 \%$ & 124,6 \\
\hline 2008 & 59,8 & $3,1 \%$ & 134,8 & 64,9 & $2,2 \%$ & 123,6 & 62,4 & $4,8 \%$ & 122,7 & 67,4 & $-0,1 \%$ & 124,5 \\
\hline 2009 & 60,9 & $1,9 \%$ & 137,2 & 66,8 & $2,9 \%$ & 127,1 & 62,7 & $0,5 \%$ & 123,3 & 70,8 & $5,0 \%$ & 130,7 \\
\hline 2010 & 62,2 & $2,1 \%$ & 140,1 & 67,5 & $1,1 \%$ & 128,6 & 64,0 & $2,0 \%$ & 125,7 & 71,1 & $0,4 \%$ & 131,3 \\
\hline 2011 & 63,1 & $1,5 \%$ & 142,1 & 68,0 & $0,7 \%$ & 129,5 & 64,3 & $0,5 \%$ & 126,4 & 71,8 & $0,9 \%$ & 132,5 \\
\hline 2012 & 63,7 & $1,0 \%$ & 143,5 & 68,7 & $0,9 \%$ & 130,7 & 64,4 & $0,1 \%$ & 126,6 & 73,0 & $1,6 \%$ & 134,7 \\
\hline 2013 & 64,4 & $1,2 \%$ & 145,2 & 69,4 & $1,1 \%$ & 132,1 & 65,2 & $1,2 \%$ & 128,1 & 73,7 & $1,0 \%$ & 136,0 \\
\hline 2014 & 63,4 & $-1,6 \%$ & 143,0 & 68,5 & $-1,3 \%$ & 130,4 & 65,2 & $0,1 \%$ & 128,2 & 71,8 & $-2,6 \%$ & 132,5 \\
\hline $2014\left(1^{\circ} \mathrm{S}\right)$ & 63,7 & -- & 143,6 & 68,9 & -- & 131,1 & 65,7 & -- & 129,1 & 72,1 & -- & 133,0 \\
\hline $2015\left(1^{\circ} \mathrm{S}\right)$ & 64,6 & $1,4 \%$ & 145,6 & 69,5 & $0,9 \%$ & 132,3 & 66,0 & $0,6 \%$ & 129,8 & 73,0 & $1,3 \%$ & 134,7 \\
\hline
\end{tabular}

Fuente: bases trimestrales de microdatos de la EPH-Continua (INDEC), $3^{\circ} \mathrm{T}$ de 2003 a $2^{\circ} \mathrm{T}$ de 2015.

Estos resultados apuntan a concluir parcialmente que, a partir de 2008, cuando la desocupación se estabiliza -continúa descendiendo, pero a un ritmo menor y con oscilaciones-, la evolución del ISMLU_PEAA quedará sujeto casi en su totalidad a las variaciones de precariedad e ingresos.

\section{ISMLU y mercado de trabajo}


Tanto el ISMLU_PEAA como el ISMLU_Ocupados se mueven en sentido inverso al de la tasa de desocupación, y en el mismo sentido en que evoluciona el PBI real per cápita (Gráfico 2). La evolución del PBI real per cápita y del ISMLU_PEAA, considerando al $2^{\circ}$ semestre de 2003 como período base, muestran una coincidencia casi exacta hasta 2007, con crecimientos acumulados cercanos al 30\%. En 2008, el ISMLU mejora más que el PBI, y al año siguiente sigue creciendo, mientras que el PBI cae. En cambio, en 2010 y 2011, el PBI se expande significativamente, mientras que el ISMLU lo hace a un ritmo menor. Finalmente, desde 2011, ambos indicadores presentan nuevamente una tendencia similar, con crecimientos acumulados hasta 2015 de algo más del $2 \%$.

Gráfico 2. Población total, Tasa de actividad, Tasa de desocupación, PBI real per cápita, Brecha entre quintiles del ingreso per cápita familiar de las personas, ISMLU_PEAA e ISMLU_Ocupados por año. Grandes aglomerados urbanos, 2003-2015. Base $100=2^{\circ} S$ de 2003

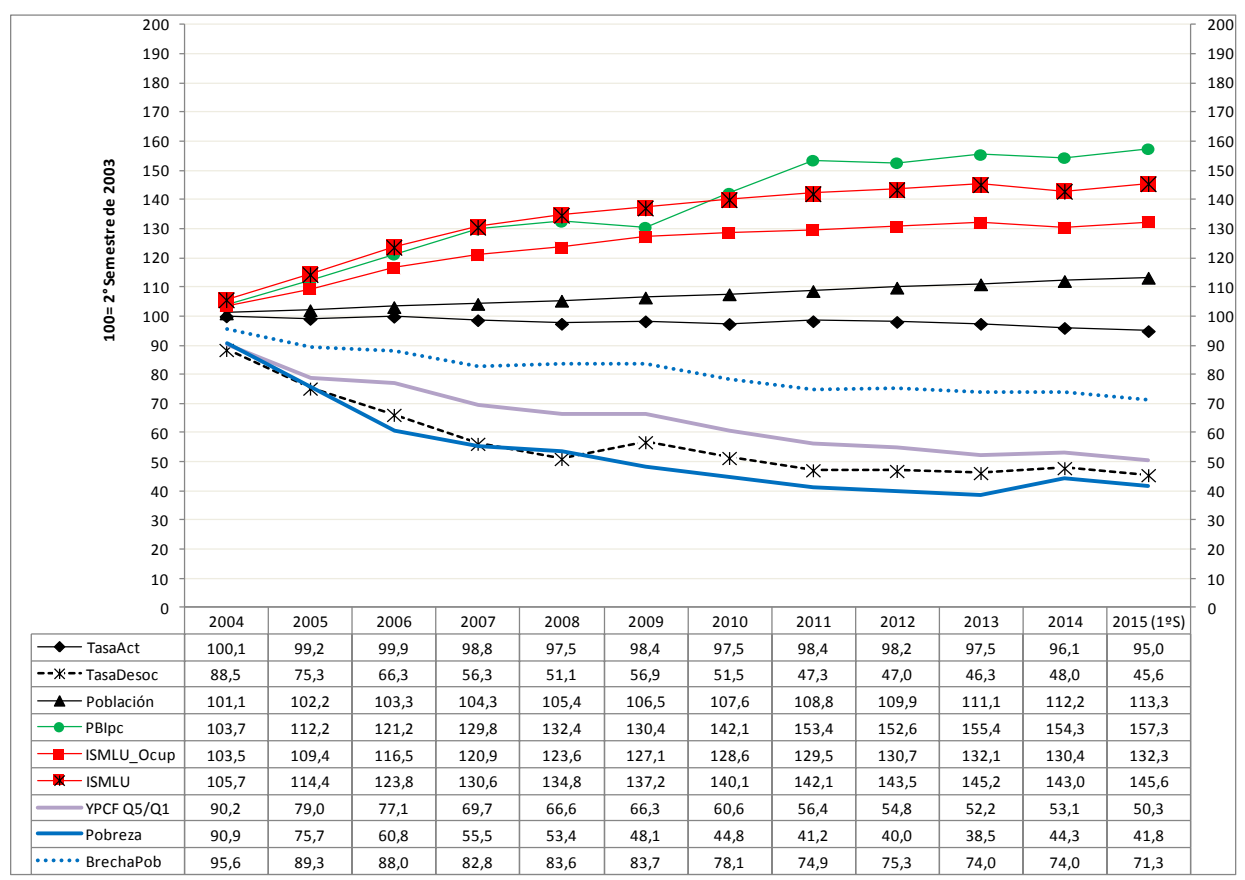

Fuente: bases trimestrales de microdatos de la EPH-Continua (INDEC), $3^{\circ} \mathrm{T}$ de 2003 a $2^{\circ} \mathrm{T}$ de 2015. INDEC: "Serie histórica por trimestre del Nivel de Actividad 1993 en adelante (en pesos de 1993)" e INDEC: "Valor Bruto de Producción-Niveles en miles de pesos a precios del año 2004". CELADE/CEPAL: "Argentina. Estimaciones y proyecciones de población a largo plazo. 1950-2100. Revisión 2015".

La brecha en la distribución del ingreso -clasificando a las personas según el ingreso per cápita familiar- también muestra una fuerte relación respecto a la situación del mercado de trabajo, disminuyendo cuando ésta última mejora. Sin embargo, en los últimos años de la serie la desigualdad continúa decreciendo -excepto en 2014mientras que la situación del mercado de trabajo muestra oscilaciones y apenas una leve mejora acumulada, por tanto, el ascenso en la distribución del ingreso 
probablemente obedezca a otros factores; esencialmente cabría suponer a la implementación de la Asignación Universal por Hijo, el plan Progresar, la nueva moratoria previsional y otras políticas de ingresos llevadas adelante por el gobierno. No obstante, la población bajo la línea de la pobreza alcanza su menor registro en 2013 $-17,6 \%$, frente a algo más del $45 \%$ en el segundo semestre de 2003 - y si bien se recupera en el primer semestre de 2015 (19,1\%) respecto a 2014 (20,3\%), continúa algo por encima de aquel año -no así la brecha de la pobreza, que presenta en 2015 el menor valor de la serie.

Finalmente, no parece existir, en este período, correlación entre el crecimiento poblacional, cercano al $1 \%$ anual, y la evolución del ISMLU. La tasa de actividad permanece prácticamente constante hasta 2012 -de alrededor del 56\% al inicio de la serie a 55\% en ese año- y a partir de allí cae hasta ubicarse en 53,2\% en el primer semestre de 2015 -parte de esta caída se explicaría por los cambios en las proyecciones de población utilizadas y por la redefinición de límites de algunos aglomerados-, aunque no puede establecerse correlación con la evolución del ISMLU.

Los resultados producto de este análisis dan cuenta de la sensibilidad e independencia del ISMLU de cambios coyunturales, pero sobre todo estructurales de las dimensiones económicas y en definitiva muestra parte de su ventaja como herramienta analítica del mercado de trabajo, que puede expresarse aislada de otras dimensiones.

\section{ISMLU y déficits}

Otra forma de analizar los resultados que surgen de la metodología implementada para el cálculo del ISMLU es a partir de la categorización ordinal del puntaje otorgado en los distintos índices. La categoría "sin déficit" incluye a quienes obtienen 100 puntos -máximo previsto- o, dicho de otra manera, a quienes logran traspasar el umbral definido como "satisfactorio". La categoría "déficit absoluto", por su parte, engloba a quienes obtienen 0 puntos. Las restantes categorías dan cuenta de las situaciones intermedias de déficit: "leve" (75 a 99,9 puntos), "moderado" (50 a 74,9 puntos), "grave" (25 a 49,9 puntos) y "muy grave" (0,1 a 24,9 puntos).

El Gráfico 3 muestra estos niveles. En el segundo semestre de 2003, el 11,4\% de la PEAA no presenta déficit en su situación laboral, mientras que el 14,9\% muestra un déficit absoluto. Ocho años después, el peso de la primera categoría más que se duplica (26,4\% sin déficit), mientras que el de la segunda se reduce a la mitad $(6,7 \%), y$ un movimiento similar se registra en la categoría de déficit muy grave. En líneas generales, las categorías de déficit moderado y grave mantienen su participación, mientras que se incrementa moderadamente el peso de la categoría déficit leve. Como se observa, la intensidad de estas modificaciones fue mayor entre 2003 y 2006 que entre 2006 y 2011. En el primer semestre de 2015, los valores son similares a los de 
2011: el 25,8\% no presenta déficit y el $6,2 \%$ se encuentra en situación de déficit absoluto.

En el caso del ISMLU_Ocupados, la evolución es similar, aunque en todos los años considerados la categoría déficit absoluto muestra un peso marginal, debido a que no se considera allí a los desocupados. Dentro de este universo, debido a la lógica de asignación de puntaje aplicada, el subíndice ingresos muestra una distribución más pareja entre las categorías. En 2003, sólo el 19,7\% de los ocupados logra trasponer el umbral fijado en un ingreso horario mensualizado superior a 1,5 CBT, proporción que se incrementa a $30,0 \%$ en 2006 y a 36,8\% en 2011 , para disminuir levemente a $36,0 \%$ en 2015. En precariedad es donde la construcción de estas categorías revela su máxima polarización, concentrando los extremos algo más del $80 \%$ del total de ocupados: mientras que en 2003 la brecha entre la proporción de ocupados sin déficit $(43,1 \%)$ y con déficit muy grave $(37,2 \%)$ en esta dimensión es de apenas 6 puntos porcentuales, en 2011 los primeros más que duplican a los segundos $(57,6 \%$ y $26,0 \%$, respectivamente). Cabe señalar que, a diferencia de lo observado en ingresos, en precariedad se observa una leve mejora entre 2011 y 2015, donde el porcentaje de ocupados sin déficit aumenta a $59,2 \%$ y quienes presentan déficit absoluto caen a $23,7 \%$. 
Gráfico 3. ISMLU_PEAA, ISMLU_Ocupados e Índices de Precariedad e Ingresos para ocupados en categorías por año. Grandes aglomerados urbanos, 2003, 2006, 2011 y 2015

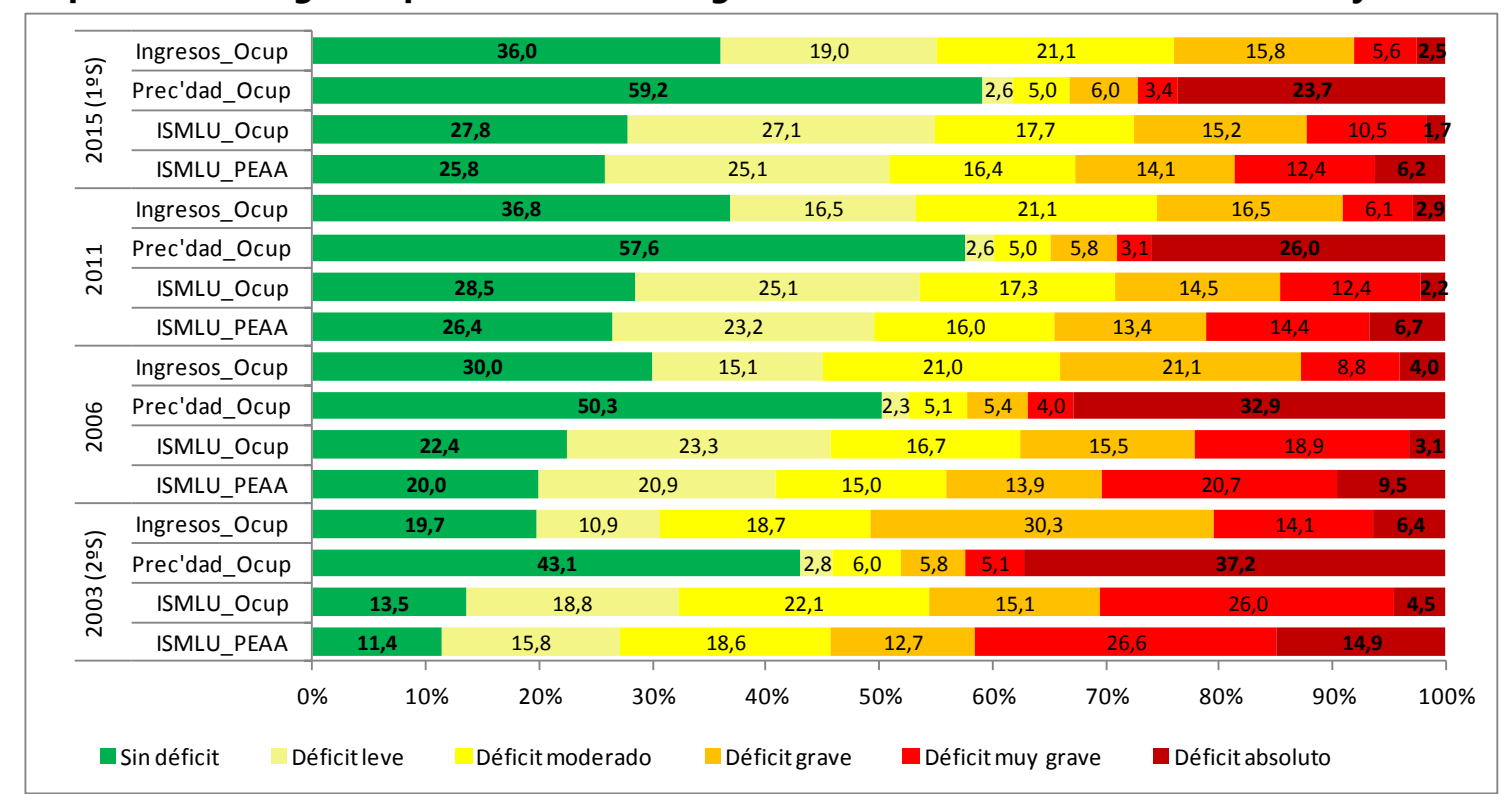

1. Fuente:bases trimestrales de microdatos de la EPH-Continua (INDEC), $3^{\circ} \mathrm{T}$ de 2003 a $2^{\circ} \mathrm{T}$ de 2015.

Existe una fuerte relación entre el mayor nivel de déficit en precariedad y la disminución del puntaje en el Índice de ingresos como se observa en el Gráfico 4, que representa la correlación entre ambas dimensiones: mientras que la misma adquiere aún mayor intensidad al considerar el nivel de déficit en ingresos y el puntaje en precariedad, a la vez que son leves las diferencias entre los años, aunque con una tendencia decreciente ${ }^{23}$.

En 2015, aquellos categorizados con déficit absoluto en precariedad presentan un Índice de ingresos promedio de 54,8 puntos, mientras que entre quienes no muestran déficit este valor asciende a 82,0 puntos. Si se considera categorialmente la dimensión ingresos, el puntaje del Índice de precariedad es de apenas 20,9 puntos entre quienes presentan déficit absoluto y alcanza 82,9 puntos entre aquellos que no muestran una situación deficitaria.

\footnotetext{
${ }^{23} \mathrm{Al}$ considerar los puntajes, la correlación ( $r$ de Pearson) entre precariedad e ingresos alcanza un valor de 0,410 en 2003, que se eleva hasta 0,485 en 2006 para caer a 0,444 en 2011 y 0,394 en 2015.
} 
Gráfico 4. Índice de Ingresos según categorías de precariedad e Índice de Precariedad según categoría de ingresos por año. Grandes aglomerados urbanos, 2003, 2006, 2011 y 2015

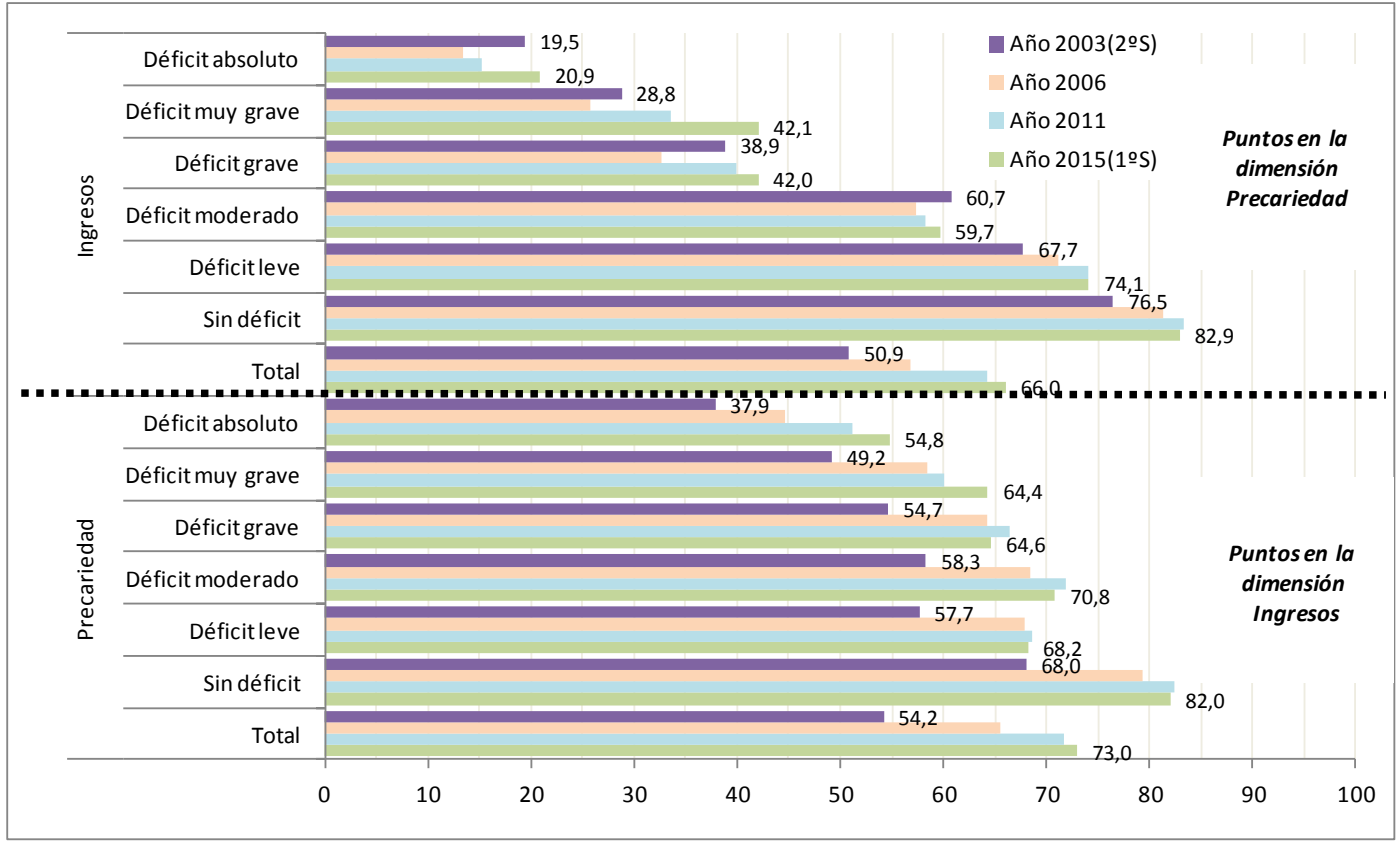

Fuente:bases trimestrales de microdatos de la EPH-Continua (INDEC), $3^{\circ} \mathrm{T}$ de 2003 a $2^{\circ} \mathrm{T}$ de 2015.

Al presentar los datos de forma agrupada, el ISMLU muestra ventajas analíticas importantes: por un lado, resume información y, por el otro, genera una estructura de niveles posibles de comparar en el tiempo.

\section{Disparidades según sexo, edad, nivel educativo y lugar de residencia}

Al considerar únicamente los momentos extremos del período de estudio-el segundo semestre de 2003 y el primer semestre de 2015-, se analizó la evolución del mercado de trabajo a través del ISMLU para distintos grupos de la población, de forma tal de dar cuenta de las posibilidades analíticas de la herramienta.

El Gráfico 5 muestra la evolución del IMSLU según sexo y edad. Los varones y los adultos -30 a 59 años en el caso de las mujeres y 30 a 64 años en el de los hombresmuestran en ambos años mejores valores en el ISMLU, pero aunque la mejora fue generalizada, dado que entre 2003 y 2015 entre las mujeres y los jóvenes de 18 a 29 años los aumentos relativos $-52 \%$ y $66 \%$, respectivamente-fueron mayores a sus contrapartes -el ISMLU creció 39\% entre los varones y 35\% entre los adultos-, tanto la brecha generacional como la brecha por sexo se reduce en el período. En 2003, el valor del ISMLU correspondiente a los hombres resultaba 15,5\% mayor al de las mujeres, mientras que en 2015 esta distancia se reduce a un tercio, llegando a sólo 5,4\% (66,4 y 63,0 puntos). La brecha por edad disminuye casi a la mitad, pasando de $70,2 \%$ a $38,1 \%$ 
entre ambos años-en 2015, el ISMLU entre los jóvenes alcanzaba a 50,9 puntos, frente a 70,3 puntos de los adultos.

\section{Gráfico 5. PEA Ampliada de 18 a 59 (mujeres)/64 años (varones). ISMLU según sexo y edad por año. Grandes aglomerados urbanos, 2003 y 2015}

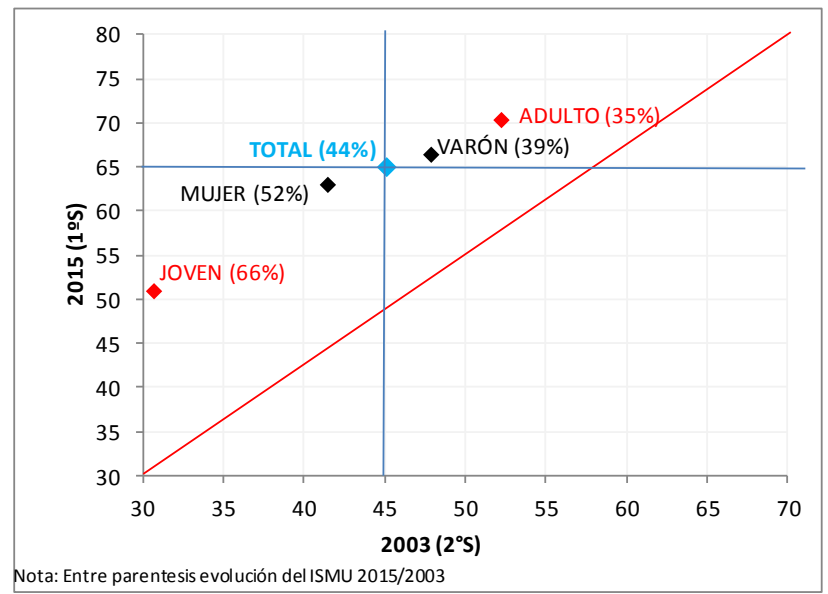

Fuente: bases trimestrales de microdatos de la EPH-Continua (INDEC), $3^{\circ} \mathrm{T}$ de 2003 a $2^{\circ} \mathrm{T}$ de 2015.

Las diferencias en el ISMLU de acuerdo al nivel educativo también se han acortado durante este período (Gráfico 6). A medida que aumenta el nivel educativo se acrecienta el valor del ISMLU, tanto en 2003 como en 2015, pero el incremento relativo adquiere más intensidad entre las personas de nivel educativo más bajo-66\% entre quienes tenían estudios primarios incompletos, frente a sólo $16 \%$ entre la población con estudios superiores completos. Así, en 2003, la brecha entre el ISMLU de quienes contaban con estudios superiores completos y quienes no habían finalizado sus estudios primarios era de casi tres veces $-73,2$ y 26,0 puntos, respectivamente- mientras que doce años después dicha distancia se acortó a dos veces ( 85,0 y 43,3 puntos). 
Gráfico 6. PEA Ampliada de 10 años y más. ISMLU según máximo nivel educativo alcanzado por año. Grandes aglomerados urbanos, 2003 y 2015

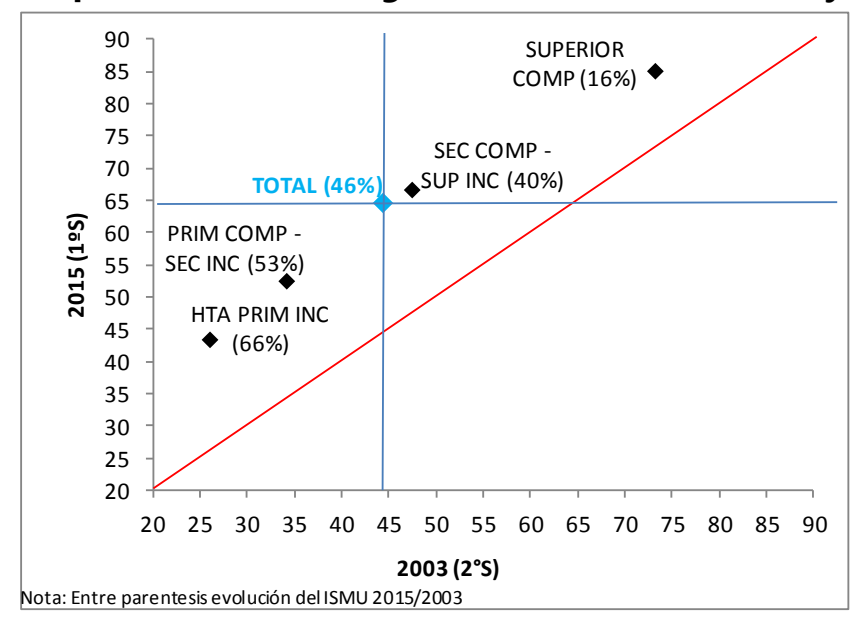

Fuente:bases trimestrales de microdatos de la EPH-Continua (INDEC), $3^{\circ} \mathrm{T}$ de 2003 a $2^{\circ} \mathrm{T}$ de 2015.

EI ISMLU revela profundas diferencias en la situación del mercado de trabajo de las diferentes regiones que conforman el país (Gráfico 7). En la ciudad de Buenos Aires y la Patagonia se registran los mejores valores del índice, tanto en 2003 como en 2015. Cuyo y la Región Pampeana -también el Gran Buenos Aires considerado como unidad, esto es, incluyendo tanto a la ciudad como a los partidos- se ubican cerca de la media del total de aglomerados urbanos. Finalmente, los Partidos del GBA, el Noreste y el Noroeste presentan las situaciones más críticas. Si bien en la ciudad de Buenos Aires y en la Patagonia el crecimiento relativo alcanzó dos terceras partes del registrado en el promedio de los grandes aglomerados, su distancia respecto a las demás regiones continúa siendo destacada. Cabe señalar que el NEA y el NOA partían en 2003 de una situación similar, muy por debajo del promedio, pero para 2015 el NEA mostraba valores apenas inferiores a la media nacional -incluso superando el registro de los Partidos del GBA-, mientras que el NOA continuaba ubicándose claramente por debajo del mismo. 
Gráfico 7. PEA Ampliada de 10 años y más. ISMLU según región de residencia por año. Grandes aglomerados urbanos, 2003 y 2015

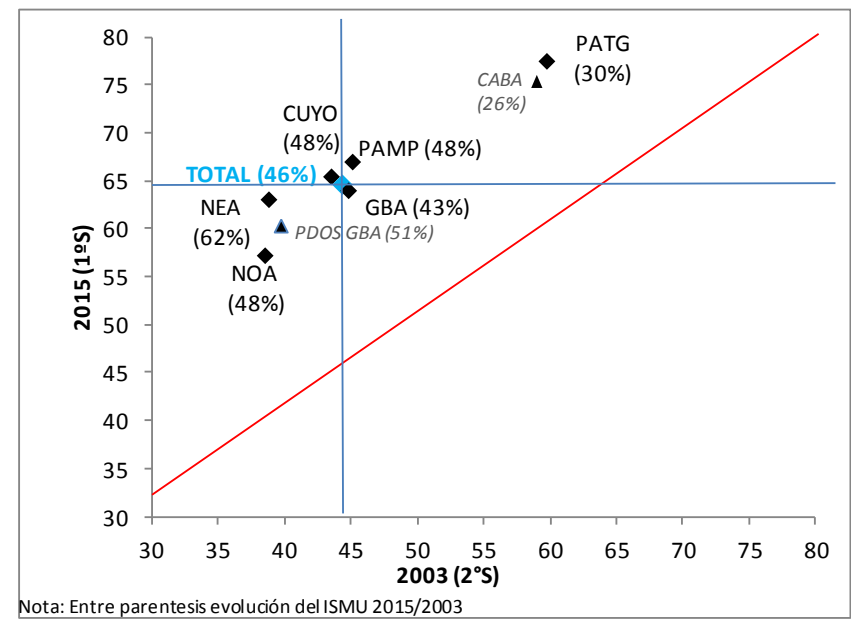

Fuente: bases trimestrales de microdatos de la EPH-Continua (INDEC), $3^{\circ} \mathrm{T}$ de 2003 a $2^{\circ} \mathrm{T}$ de 2015.

\section{Conclusiones}

El análisis del mercado de trabajo urbano en Argentina en los últimos 12 años utilizando el IMSLU mostró las posibilidades de aplicación de una herramienta sintética y a la vez multidimensional, tanto para monitorear la evolución temporal del mercado de trabajo como para dar cuenta de las diferencias entre grupos sociales para insertarse plenamente en el mismo.

Al partir de un momento de inicio bajo un contexto económico y social crítico, el IMSLU pasa de 44,4 puntos en el segundo semestre de 2003 a 64,6 puntos en el primer semestre de 2015. En otras palabras, en estos 12 años se ha reducido en casi dos quintas partes -37,5\%- la distancia entre la situación original y la situación "ideal" que subyace a la construcción metodológica del IMSLU y que puede definirse como una situación de pleno empleo, con ocupaciones estables y cubiertas por la seguridad social, además de ingresos laborales mensualizados ubicados por encima del umbral del valor de una canasta y media de pobreza para un hogar tipo.

Sin embargo, esta evolución no fue lineal y el ISMLU permite marcar tres etapas bien diferenciadas: en el período 2003-2007 se observan mejoras de gran intensidad, fundamentalmente por la disminución de la desocupación y el aumento de los ingresos reales, en tanto que el aporte de la dimensión precariedad es también significativo, pero de menor cuantía. Hasta 2011, las mejoras continúan, aunque con menor intensidad, y son explicadas, en forma no lineal, por cambios en los tres componentes. Desde 2011 a 2015, el ISMLU muestra valores oscilantes, aunque el primer semestre de 2015 registra, por escasa diferencia, los mejores valores de la serie y una recuperación respecto a la caída de 2014 - la única observada en toda la serie y explicada fundamentalmente por la baja en ingresos, producto de una inflación de niveles 
claramente superior a la de los años previos. Cabe señalar que, en los últimos años, las mejoras en el ISMLU obedecen más a la leve disminución de la desocupación y a la mejora en precariedad que a la evolución de ingresos.

El creciente valor del IMSLU a lo largo del período estuvo acompañado por una importante disminución de la desigualdad. Si bien las distintas subpoblaciones siguen el patrón general positivo, se observa que la intensidad de este progreso fue mayor en aquellos grupos que al inicio de la serie presentan las situaciones más desfavorables, lo cual redunda en una menor desigualdad relativa hacia el final del período considerado, aunque con disímil intensidad según el caso, considerando el sexo, la edad, el nivel educativo y la región de residencia.

\section{Perspectivas}

El análisis propuesto puede profundizarse desde diversas aristas: a partir de considerar la evolución y/o las diferencias en las dos dimensiones (precariedad, ingresos) entre diversos grupos de la población, así como de la construcción de grupos específicos-cruzando dos o más variables de las aquí presentadas o bien introduciendo otras nuevas-, siguiendo con mayor detalle los cambios a través del tiempo y también utilizando categorizaciones ordinales.

El nudo gordiano de la metodología del ISMLU es, sin dudas, la estructura de ponderaciones y sobre esto es necesario realizar varias consideraciones. En primer lugar, el ejercicio se presentó como una primera exploración, como un punto de partida y no de llegada; por tanto, todas las decisiones tomadas están sujetas al debate porque en última instancia eso es lo que buscan incentivar. Segundo, tanto las dimensiones como los indicadores se han construido con, y sobre la base de las limitaciones de la información disponible en la EPH, referente empírico sobre el cual se asienta -y del que, en sentido estricto, resulta inseparable- el ISMLU; por tanto, en algunos casos algunas aproximaciones resultan menos directas que en otras -por ejemplo, la adscripción a la seguridad social se puede corroborar de manera directa en el caso de los asalariados, pero no para los cuentapropistas y patrones para quienes no existe información sobre la realización de aportes jubilatorios- $y$, a la vez, existen muchos temas de suma relevancia que ni siquiera es posible abordar -bienestar subjetivo, riesgos de la salud, etc.- con la información disponible en la EPH.

En tercer lugar, la operacionalización de la dimensión precariedad requiere muchas más mediaciones que la dimensión ingresos. En este caso, la línea de pobreza -ingresos que una familia tipo necesita para no ser pobre- constituye un umbral más que razonablemente lógico, y si aquí se ha considerado 1,5 CBT en lugar de simplemente una CBT para hogar tipo, es a modo de "precaución conservadora" porque la CBTINDEC, más allá de su valorización, adolece de serias deficiencias metodológicas construcción sobre la base de parámetros de consumo de 30 años atrás, estimación indirecta del valor de los componentes no alimentarios, no contemplación de 
economías de escala al interior del hogar, no distinción de hogares según características básicas como el hecho de ser o no inquilinos de la vivienda, etc.

Finalmente, y en otro orden, el ISMLU no considera la tasa de actividad; si bien en el corto plazo los cambios en este indicador no suelen ser bruscos, y por tanto no afectarían la comparabilidad longitudinal del índice -aunque requiere especial cuidado la caída registrada a partir de 2013-, merece discusión si esto mismo podría sostenerse en caso de ampliar el período analizado, por ejemplo, a la década del noventa.

Más allá de estas precauciones y limitaciones, puede aventurarse que modificaciones al respecto no afectarían drásticamente los resultados obtenidos con el ISMLU para el período analizado, al menos en lo que hace a la tendencia e intensidad de la evolución temporal y a las diferencias entre distintos grupos sociales.

\section{Anexo}

Tabla 2. Valor trimestral de la Canasta Básica Total para un hogar Tipo del Gran Buenos Aires, $2^{\circ}$ trimestre $2003-2^{\circ}$ trimestre 2016

\begin{tabular}{|c|c|c|c|c|c|}
\hline $\begin{array}{l}\text { Trimestre } \\
\text { (AAAAT) }\end{array}$ & $\begin{array}{c}\text { CBT } \\
\text { Hogar } \\
\text { Tipo }\end{array}$ & $\begin{array}{l}\text { Var. Trim. } \\
\text { Ant (\%) }\end{array}$ & $\begin{array}{c}\text { Var. } \\
\text { Interan. }\end{array}$ & 1,5CBT_HT & YHRef \\
\hline 20033 & 701 & $-2,1$ & 3,8 & 1052 & 5,43 \\
\hline 20034 & 702 & 0,2 & $-1,8$ & 1053 & 5,44 \\
\hline 20041 & 717 & 2,1 & $-1,1$ & 1075 & 5,56 \\
\hline 20042 & 720 & 0,5 & 0,5 & 1080 & 5,58 \\
\hline 20043 & 726 & 0,8 & 3,6 & 1089 & 5,63 \\
\hline 20044 & 735 & 1,2 & 4,7 & 1103 & 5,70 \\
\hline 20051 & 749 & 1,8 & 4,5 & 1123 & 5,80 \\
\hline 20052 & 772 & 3,2 & 7,3 & 1159 & 5,99 \\
\hline 20053 & 786 & 1,7 & 8,2 & 1178 & 6,09 \\
\hline 20054 & 813 & 3,5 & 10,6 & 1220 & 6,30 \\
\hline 20061 & 841 & 3,5 & 12,4 & 1262 & 6,52 \\
\hline 20062 & 857 & 1,8 & 10,9 & 1285 & 6,64 \\
\hline 20063 & 859 & 0,3 & 9,3 & 1289 & 6,66 \\
\hline 20064 & 870 & 1,3 & 7,0 & 1305 & 6,74 \\
\hline 20071 & 905 & 4,0 & 7,6 & 1358 & 7,02 \\
\hline 20072 & 957 & 5,7 & 11,7 & 1435 & 7,42 \\
\hline 20073 & 1059 & 10,7 & 23,3 & 1589 & 8,21 \\
\hline 20074 & 1181 & 11,4 & 35,7 & 1771 & 9,15 \\
\hline 20081 & 1204 & 2,0 & 33,0 & 1806 & 9,33 \\
\hline 20082 & 1356 & 12,7 & 41,8 & 2035 & 10,52 \\
\hline 20083 & 1411 & 4,0 & 33,2 & 2116 & 10,94 \\
\hline
\end{tabular}




\begin{tabular}{|c|c|c|c|c|c|}
\hline 20084 & 1412 & 0,1 & 19,6 & 2118 & 10,95 \\
\hline 20091 & 1435 & 1,7 & 19,3 & 2153 & 11,13 \\
\hline 20092 & 1482 & 3,2 & 9,3 & 2223 & 11,49 \\
\hline 20093 & 1531 & 3,3 & 8,6 & 2297 & 11,87 \\
\hline 20094 & 1613 & 5,3 & 14,2 & 2420 & 12,51 \\
\hline 20101 & 1691 & 4,8 & 17,8 & 2536 & 13,10 \\
\hline 20102 & 1831 & 8,3 & 23,5 & 2746 & 14,19 \\
\hline 20103 & 1865 & 1,9 & 21,8 & 2797 & 14,46 \\
\hline 20104 & 2077 & 11,4 & 28,8 & 3116 & 16,10 \\
\hline 20111 & 2231 & 7,4 & 31,9 & 3346 & 17,29 \\
\hline 20112 & 2337 & 4,8 & 27,7 & 3506 & 18,12 \\
\hline 20113 & 2439 & 4,4 & 30,8 & 3659 & 18,91 \\
\hline 20114 & 2551 & 4,6 & 22,8 & 3826 & 19,77 \\
\hline 20121 & 2706 & 6,1 & 21,3 & 4058 & 20,97 \\
\hline 20122 & 2913 & 7,7 & 24,6 & 4370 & 22,58 \\
\hline 20123 & 3105 & 6,6 & 27,3 & 4658 & 24,07 \\
\hline 20124 & 3320 & 6,9 & 30,2 & 4980 & 25,74 \\
\hline 20131 & 3474 & 4,6 & 28,4 & 5211 & 26,93 \\
\hline 20132 & 3634 & 4,6 & 24,8 & 5451 & 28,17 \\
\hline 20133 & 3875 & 6,6 & 24,8 & 5812 & 30,04 \\
\hline 20134 & 4178 & 7,8 & 25,8 & 6267 & 32,39 \\
\hline 20141 & 4542 & 8,7 & 30,7 & 6812 & 35,21 \\
\hline 20142 & 5068 & 11,6 & 39,5 & 7602 & 39,29 \\
\hline 20143 & 5420 & 6,9 & 39,9 & 8130 & 42,01 \\
\hline 20144 & 5770 & 6,5 & 38,1 & 8655 & 44,73 \\
\hline 20151 & 6052 & 4,9 & 33,3 & 9078 & 46,91 \\
\hline 20152 & 6412 & 6,0 & 26,5 & 9618 & 49,71 \\
\hline 20153 & 6797 & 6,0 & 25,4 & 10196 & 52,69 \\
\hline 20154 & 7177 & 5,6 & 24,4 & 10765 & 55,64 \\
\hline 20161 & 7820 & 9,0 & 29,2 & 11731 & 60,62 \\
\hline 20162 & 8879 & 13,5 & 38,5 & 13318 & 68,83 \\
\hline
\end{tabular}

Elaboración propia sobre labase de la CBT-INDEC (hasta $4^{\circ} \mathrm{T}$ 2006), CBT Ciudad de

Paraná-DPE Entre Ríos (hasta 4T 2010) y CT-Hogar 1 del Sistema de Canastas de Consumo de la DGEyC-CABA -hasta 2012 se utilizó el IPC Congreso para modelizar las variaciones mensuales de la $\mathrm{CT}$, pues sólo se contaba con el dato de noviembre de cada año.

\section{Bibliografía}

Alkire, S. y Foster, J. (2007).Recuento y medición multidimensional de la pobreza. EnOPHI Working Paper 7. UK: University of Oxford. 
Alkire y Robles (2015). Multidimensional Poverty Index-2015: Brief Methodological Note and Results.UK: University of Oxford.

Beccaria, L. y Maurizio, R. (2008). Mercado de trabajo y distribución personal del ingreso. En J. Lindenboim (ed.) Trabajo, ingresos y políticas en Argentina. Contribuciones para pensar el siglo XXI. Buenos Aires: Eudeba.

Beccaria, L. (2010). Enfoque de derechos y pobreza multidimensional. EnSeminario Internacional Medición multidimensional de la pobreza en América Latina, CEPAL, Santiago de Chile.

Becker, G. S. (1983). Capital humano: un análisis teórico y empírico referido fundamentalmente a la educación. Madrid: Alianza.

CENDA (2004). Índice Global de Condiciones de Trabajo. El trabajo en la Argentina. Condiciones y Perspectivas, Informe N¹,otoño. Disponible en:http://cenda.org.ar/.

CEPAL (2014). Panorama Social de América Latina, 2014, Santiago de Chile, CEPALONU.

CEPAL-UNICEF (2010). Pobreza infantil en América Latina y el Caribe, Santiago de Chile.

CEPED (2010). Evolución de las condiciones de trabajo y sus límites: el caso de los aglomerados de la Provincia de Buenos Aires. En Concurso Ministerio de Trabajo de la Provincia de Buenos Aires.

Chávez Molina, E. y Sacco, N. (2015). Reconfiguraciones en la estructura social: dos décadas de cambios en los procesos distributivos. Análisis del GBA según en el clasificador de clases ocupacionales basado en la heterogeneidad estructural 19922013. En J. Lindenboim y A. Salvia (eds.) Hora de balance: proceso de acumulación, mercado de trabajo y bienestar. Argentina, 2002-2014. Ciudad Autónoma de Buenos Aires: Eudeba.

CONEVAL (2012).Metodología para la medición multidimensional de la pobreza en México, México. Disponible en http://www.coneval.org.mx.

Damill y Frenkel, R. (2013). La economía argentina bajo los Kirchner: una historia de dos lustros. Buenos Aires: ITF.

Fernández, D. D.; Fernández, C. I. y Heras, R. L. (2009). La calidad del empleo en un contexto regional, con especial referencia a la Comunidad de Madrid. Documentos de Trabajo. Disponible en:http://www.iaes.es/

Green, F. (2006). Demanding Work. The paradox of job quality in the affluent economy. Princeton and Oxford: Princeton University Press.

Groisman, F.; Vergara, A. y Calero, A. (2011). Cambios en la informalidad en el mercado de trabajo argentino (2004-2010). Ponencia presentada en $10^{\circ}$ Congreso Nacional de Estudios del Trabajo, Facultad de Ciencias Económicas de la UBA, Buenos Aires, Argentina.

INDEC (2002). Metodología de transición en incidencia de la pobreza y de la indigencia en los aglomerados urbanos. Disponible en:http://www.indec.mecon.ar/ 
--- (2003). Acerca del método utilizado para la medición de la pobreza en Argentina. Disponible en:http://www.indec.mecon.ar/

--- (2011). Encuesta Permanente de Hogares. Conceptos de Condición de Actividad, Subocupación Horaria y Categoría Ocupacional. Disponible en:http://www.indec.mecon.ar/

Jencks, C.; Perman, L. y Rainwater, L. (1988). What Is a Good Job? A New Measure of Labor-Market Success. EnAmerican Journal of Sociology, 93, 6, 1322-1357.

Kessler, G. (2014). Controversias sobre la desigualdad: Argentina, 2003-2013. Buenos Aires, Argentina: Fondo de Cultura Económica.

Kostzer, D.; Perrot, B.; Schachtel, L. y Villafañe, S. (2005). Índice de fragilidad laboral: un análisis geográfico comparado del empleo y el trabajo a partir del EPH. Buenos Aires: Ministerio de Trabajo de la Nación.

Layard, R. (2005). Happiness. Lessons from a New Science. London: Allen Lane.

Lindenboim, J. y Beccaria, L. (2008). Trabajo, ingresos y políticas en Argentina: contribuciones para pensar el siglo XXI ( $1^{\mathrm{a}}$ ed.). Ciudad de Buenos Aires: Eudeba.

Martel, J.P. y Dupuis, G. (2006). Quality of Work Life: Theoretical and Methodological Problems, and Presentation of a New Model and Measuring Instrument. [Article]. EnSocial Indicators Research, 77, 2, 333-368. doi: 10.1007/s11205-004-5368-4.

Monza, A. (1993). La situación ocupacional argentina: diagnóstico y perspectivas.Desigualdad y exclusión: desafíos para la política social en la Argentina de fin de siglo. Buenos Aires: UNICEF : Losada.

Muñoz de Bustillo Llorente, R. y Fernández Macías, E. (2005). Job satisfaction as an indicator of the quality of work. EnJournal of Socio-Economics, 34, 5, 656-673. doi: 10.1016/j.socec.2005.07.027.

Neffa, J. C. (1995). Las condiciones y medio ambiente de trabajo (CyMAT). Disponible en:http://www.ceil-piette.gov.ar/pub.html

Pok, C. (1992). Precariedad laboral: personificaciones sociales en la frontera de la estructura del empleo. Documento presentado en el Primer Congreso Nacional de Estudios del Trabajo (Buenos Aires) y en el Seminario Interamericano Medición del Sector Informal, OEA, 26-28 de agosto, Lima.

Rovira, E. C.; Hoz, E. V.; Canals, C. S. y Alcaide, M. F. (2012). Construcción de un Índice de Calidad Ocupacional (ICO) para el análisis de la inserción profesional de los graduados universitarios. EnRevista de Educación, 357. Disponible en:http://www.revistaeducacion.educacion.es/

Royuela, V.; López-Tamayo, J. y Suriñach, J. (2009). Results of a Quality of Work Life Index in Spain. A Comparison of Survey Results and Aggregate Social Indicators. [Article].EnSocial Indicators Research, 90, 2, 225-241. doi: 10.1007/s11205-008-92543.

Salvia, A. (2015). Reflexiones finales: Algunas claves para entender el subdesarrollo persistente argentino. En J. Lindenboim y A. Salvia (eds.) Hora de balance: proceso de 
De Prácticas y discursos/ Universidad Nacional del Nordeste/ Centro de Estudios Sociales

acumulación, mercado de trabajo y bienestar. Argentina, 2002-2014. Ciudad Autónoma de Buenos Aires: Eudeba.

Schorr, M. y Wainer, A. (2011). Posconvertibilidad:¿resurgimiento de un capitalismo nacional o continuidad de la extranjerización económica? Un análisis a partir del panel de las grandes empresas de argentina. EnIX Jornadas de Sociología de la UBA. 